\title{
The energetics of small molecules binding with nucleic acids
}

\author{
Viktor V. Kostjukov ${ }^{\mathrm{a}}$, Maxim P. Evstigneev ${ }^{\mathrm{a}, \mathrm{b}, *}$ \\ ${ }^{a}$ Department of Physics, Sevastopol State University, Sevastopol 299053, Russian Federation \\ ${ }^{\mathrm{b}}$ Laboratory of Organic Synthesis and NMR Spectroscopy, Belgorod State University, Belgorod 308015, Russian Federation
}

\section{A R T I C L E I N F O}

\section{Article history:}

Received 29 May 2019

Received in revised form 30 July 2019

Accepted 1 August 2019

Available online 3 August 2019

\section{Keywords:}

Energy analysis

Energy decomposition

Thermodynamics of complexation

Ligand-nucleic acid complexation

\begin{abstract}
A B S T R A C T
The present review deals with energy analysis of ligand binding with nucleic acids in terms of the energy contribution from various physical factors making up the net Gibbs energy change of the complexation reaction measured in experiment. The general goal of such analysis may be formulated in a form of answering two questions, viz. "What physical factors and to what extent stabilize/destabilize ligand-NA complexes?' and 'What physical factor most highly correlates with the parameter of interest?' (e.g. the complexation constant or biological activity or else). Three groups of interactions were considered, viz. ligand-DNA intercalation, ligand-DNA minor groove binding and non-intercalative ligand binding to RNA aptamers. The general patterns in distribution of energy over various energy terms were discussed in terms of correlation with the ligand structure, binding affinity and biological activity. It was concluded, that the energy analysis, as a part of thermodynamic method of investigation of ligand-nucleic acid interactions, may provide an extension to scientific background of the strategy of rational design of DNA/RNA targeting drugs.
\end{abstract}

(c) 2019 Elsevier Ltd.

\section{Contents}

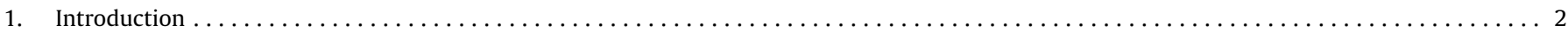

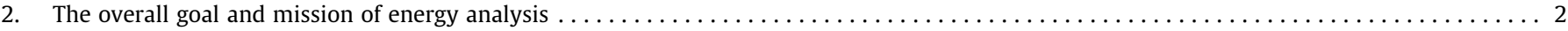

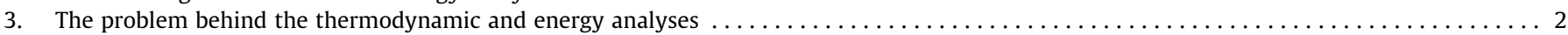

4. Brief survey of the methods of computation of energy terms related to contribution of particular physical factors to binding energetics ...... 3

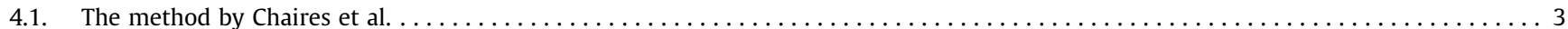

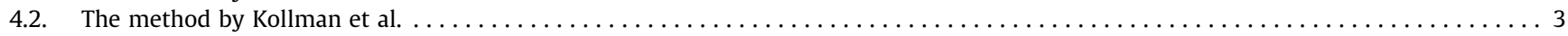

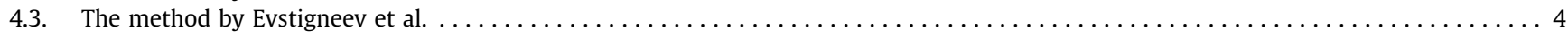

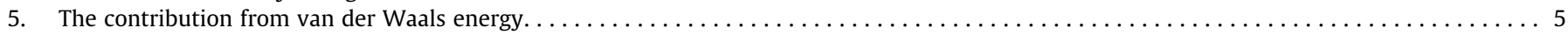

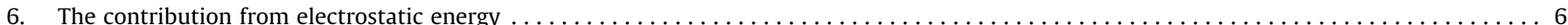

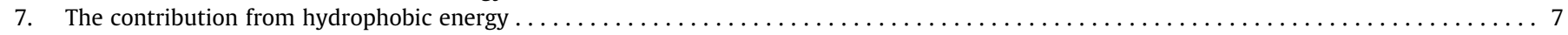

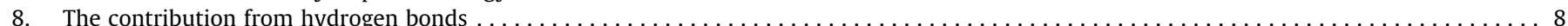

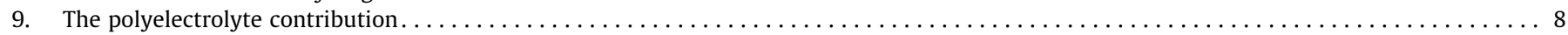

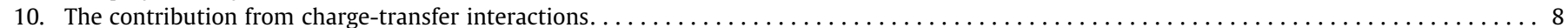

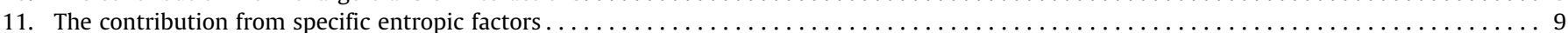

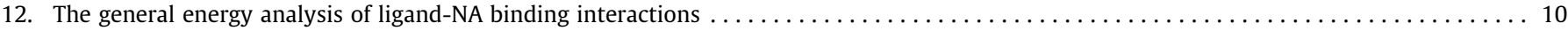

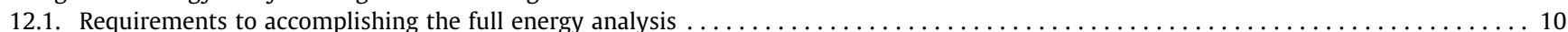

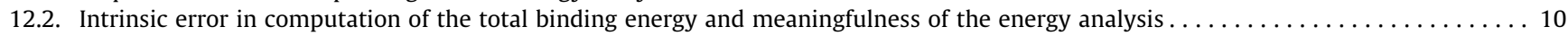

12.3. What physical factors and to what extent stabilize/destabilize complexes of small molecules with NA? $\ldots \ldots \ldots \ldots \ldots \ldots \ldots \ldots$

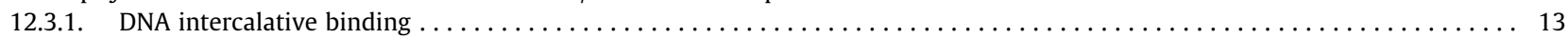

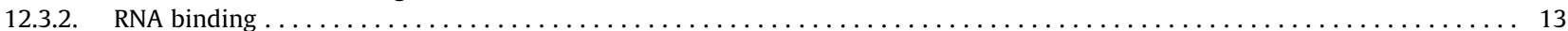

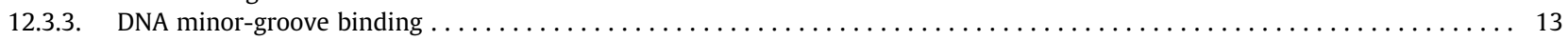

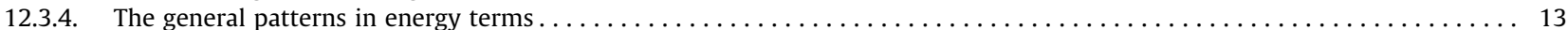

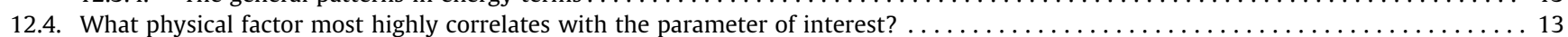

\footnotetext{
* Corresponding author at: Laboratory of Organic Synthesis and NMR Spectroscopy, Belgorod State University, Russian Federation.

E-mail address: max_evstigneev@mail.ru (M.P. Evstigneev).
} 


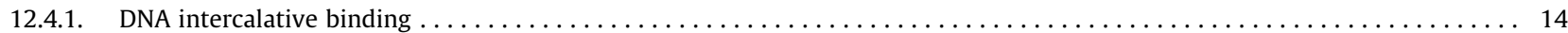

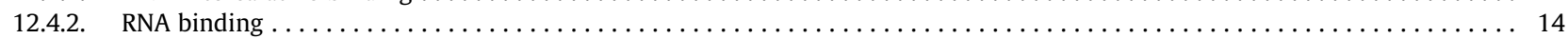

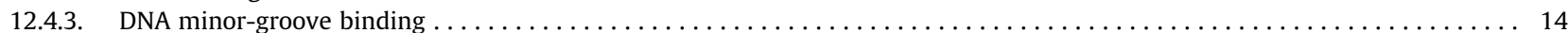

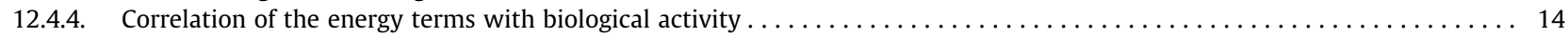

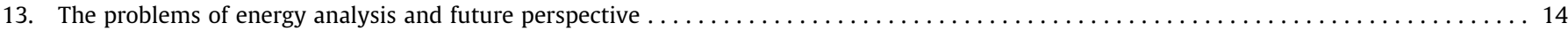

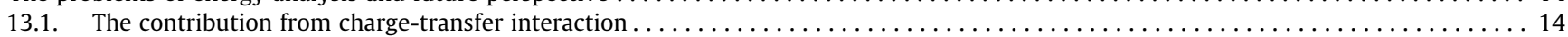

13.2. Solution of the energy decomposition task for enthalpy, entropy and heat capacity changes. . . . . . . . . . . . . . . . . $14 \ldots \ldots \ldots$

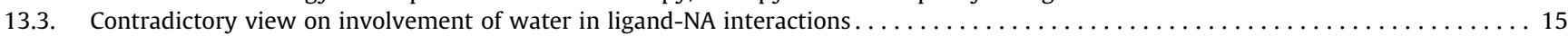

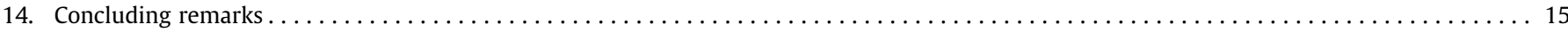

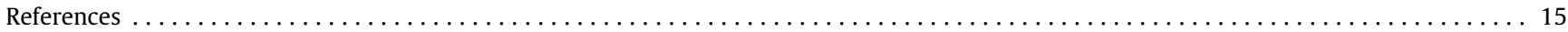

\section{Introduction}

Nucleic acids (NA) exist in cells of all living organisms and execute important functions such as storage, transmittance and realization of genetic information. Due to crucial role played by NA in living organisms, of great importance is the development of means for their targeted affection with an aim to achieve the desired medico-biological effect. One of the most effective approaches in creation such action is the binding of NA by small biologically active molecules (to be further referred to as the ligands), and the thermodynamics of the binding reactions lies behind an understanding of the ligand complexation-induced biological action [1-3].

Systematic investigations of the structure and thermodynamics of ligand binding with NA count its history from Watson and Crick discovery of the structure of DNA double helix in 1953 and have to date been thoroughly reviewed (e.g. [1,4-6]). General thermodynamic analysis of ligand-NA interactions is commonly based on experimental determination of Gibbs energy $(\Delta G)$, enthalpy $(\Delta H)$, entropy $(\Delta S)$ and heat capacity $\left(\Delta C_{p}\right)$ changes in binding reaction with further comparative analysis within the specially-selected set of ligands possessing some structural or physico-chemical similarity. In very first approximation the thermodynamic parameters of binding may be correlated with biological response (for reviews see $[4,7-9]$. The typical examples are the semi-synthetic antibiotic Novantrone (an anthracycline derivative), which is widely used in the treatment of leukaemia [10], and bis-doxorubicin (a doxorubicin derivative), which exhibits activity against multidrugresistant tumour cells [11]. It follows that a manipulation by the parameters of drug-DNA binding by means of directed chemical synthesis of the drug molecules may potentially lead to creation of new drugs, and provides scientific basis for rational drug design. However, a link between the thermodynamic potentials, $\Delta G, \Delta H$, $\Delta S, \Delta C_{p}$, and the exact structure of the ligand to be synthesized is not generally known. Partial solution of this problem may be accomplished within the framework of energy analysis, whose objective is to determine the 'energy profile' of the binding reaction, i.e. to find out what physical factors (van der Waals, electrostatic, hydrophobic etc) and to what extent contribute to experimentally measured thermodynamic potentials [6,12-15]. Even more important outcome would be the determination of correlation of particular energy terms, related to particular physical factors, and some biological parameter (e.g. $\left.\mathrm{IC}_{50}\right)$. Once found, it may give an idea of which physical factor should be targeted in first instance when optimizing drug affinity to DNA in rational drug design $[5,7,12,16]$. In terms of synthesis it gives a clue on what type of atomic group should be added to the structure of ligand in order to get desired energy profile of binding.

Historically the energy analysis of ligand-bioreceptor bindings started from investigation of protein-involving reactions, and the energetics of ligand-protein interactions is now characterized much better than ligand-NA interactions (e.g. $[2,12,16-18])$. The set of systematic reviews on the energetics of ligand binding with NA was given in $[5,6,15,19,20]$, being, however, limited to a single methodology of energy analysis and providing no insight into the contribution of enthalpic factors such as van der Waals, electrostatic and H-bonding. During the past decade numerous papers appeared providing broader understanding of the topic.

The aim of this paper is to create general view on the current state-of-the-art of energy analysis of ligand binding with DNA and RNA. The focus of the review will be the general patterns of energy distribution over various energy terms, leaving aside the specificity of binding in each particular case. The discussion will be confined to three most systematically studied (in terms of energy analysis) groups of ligand-NA interactions, viz. DNA intercalation, DNA minor-groove binding (MGB) and non-intercalative ligand binding to RNA aptamers.

\section{The overall goal and mission of energy analysis}

The general tasks of energy analysis, implicitly or explicitly stated in majority of published works in this field of research (e.g. Refs. $[3,14,15])$, may be formulated in the form of answering the two principal questions:

1. 'What physical factors and to what extent stabilize/destabilize ligand-NA complexes?' and

2. 'What physical factor most highly correlates with the parameter of interest?' (e.g. the complexation constant or biological activity or else).

The method of energy analysis would include the computation of various terms of the net Gibbs energy change on ligand-NA complexation and their analysis with respect to answering the two main questions given above. It is considered that solution of all these tasks may significantly improve scientific basis, and, probably, give breakthrough in the field of rational drug design, resulting in creating of new drugs with given thermodynamic profile of binding with bioreceptor (i.e. managing the biological outcome via manipulating the contribution of specific energy terms). This strategy, in fact, has been formulated in general terms more than 20 years ago [19], although the most extensive embodying of this idea has become possible only in recent years due to rapid development of power of computing.

\section{The problem behind the thermodynamic and energy analyses}

It has long been recognized that experimentally measured thermodynamic parameters, $\Delta G, \Delta H, \Delta S$ and $\Delta C_{\mathrm{p}}$, are made up of the sum of contributions from various types of physical interactions (see Refs. [14,15] and references therein), viz. van der Waals, electrostatic, hydrophobic etc: 
$\Delta G($ or $\Delta H, \Delta S)=\sum_{i} \Delta G_{i}\left(\right.$ or $\left.\Delta H_{i}, \Delta S_{i}\right)$

where $\Delta G_{i}$ (or $\Delta H_{i}, \Delta S_{i}$ ) stands for the contribution of the $i$-th physical factor to $\Delta G$ (or $\Delta H, \Delta S$ ) and to be further referred to as the 'energy terms'.

Any modification in the structure of a ligand in general case is likely to lead to unpredictable change in values of the energy components in Eq. (1) and the effect of their summation in Eq. (1) may change the $\Delta G|\Delta H| \Delta S / \Delta C_{p}$ or even leave them unchanged. It follows that direct comparison of experimentally-measured thermodynamic potentials for different ligands (i.e. the basis of general thermodynamic analysis) is unlikely to be very meaningful and may even lead to erroneous conclusions. A common manifestation of that problem is encountered in the enthalpy-entropy compensation for binding processes in aqueous media [21-24], as well as the longexisting discussion in the literature on what forces (van der Waals, electrostatic or hydrophobic) or types of interactions (solutesolvent or solute-solute) dominate in binding reactions (see [22,25-27]), which makes a thermodynamic analysis intrinsically ambiguous. Nevertheless, greater understanding of the thermodynamics of drug-DNA binding processes can be achieved if the task of energy partitioning (also known as energy parsing or energy decomposition) is solved. Solution of this task is pre-requisite to any energy analysis and was first systematically pioneered by Chaires et al. with respect to ligand-NA complexations $[6,20,28]$. It requires an independent calculation of the energy components in Eq. (1) and comparison of the results to the experimentallymeasured total Gibbs energy. However, there are, at least, two fundamental problems behind any attempt to parse experimentallymeasured thermodynamic quantities $\Delta G, \Delta H, \Delta S$, viz.

(i) it is not possible to measure independently the contribution of specific energy term to the total binding energies, and

(ii) the energy terms contributing to non-covalent binding energetics in solution are all originate from fundamental electromagnetic interaction and the follow up question arises whether the decomposition of $\Delta G, \Delta H, \Delta S$ is physically meaningful [22].

Although the (ii) problem seems as already being theoretically addressed $[22,29]$, both problems will always lie behind any thermodynamic and energy analyses of binding reactions in solution.

\section{Brief survey of the methods of computation of energy terms related to contribution of particular physical factors to binding energetics}

To date the methods of computation of the net Gibbs energy change for the reactions of complexation of various ligand molecules with nucleic acids have been elaborated in detail and extensively reviewed in scientific literature. These methods include docking methods (such as DrugScore ${ }^{\text {RNA }}[30,31]$, hydropathic analysis [3234], genetic [35] and Monte-Carlo [36] algorithms) and/or routine molecular simulation approaches (such as energy perturbation methods [38-40] or molecular mechanics methods [41]), which, in majority of cases, do not decompose the resultant net Gibbs energy onto energy terms. We shall further focus our attention specifically on the methods aiming to compute the net Gibbs energy change as a sum of independently calculated energy terms related to particular physical factors (i.e. the energy decomposition task).

The most systematic solution of the energy decomposition task for the group of ligand-NA binding reactions has been accomplished within the three main approaches, suggested by Chaires et al., Kollman et al., and Evstigneev et al. The key criterion of the success of the methods of energy decomposition would be the coincidence of theoretically calculated, $\Delta G_{\text {total }}$, and experimentally measured, $\Delta G_{\text {exp }}$, Gibbs energy changes for structurally different ligand molecules. Under the above-mentioned limitation of inability to measure the contribution of particular physical factor to binding energetics, this criterion remains the only condition making the analysis of separate energy terms physically meaningful.

\subsection{The method by Chaires et al.}

The most simple and intuitively clear approach for energy decomposition was suggested by Chaires et al., who accomplished energy analyses of DNA binding reactions for ligands different by structure and type, viz. DNA mono- $[28,42]$ and bis- $[28,43]$ intercalators, and DNA minor groove binders [28,44]. The net Gibbs energy change on complexation is given as a sum of the following terms

$\Delta G_{\text {total }}=\Delta G_{\text {conf }}+\Delta G_{\mathrm{r}+\mathrm{t}}+\Delta G_{\mathrm{pe}}+\Delta G_{\text {hyd }}+\Delta G_{\mathrm{mol}}$,

where $\Delta G_{\text {conf }}$ is a contribution from conformational changes of the ligand and DNA; $\Delta G_{\mathrm{r}+\mathrm{t}}$ is energetic equivalent of the loss of translational and rotational degrees of freedom; $\Delta G_{\text {pe }}$ is a polyelectrolyte contribution; $\Delta G_{\mathrm{mol}}$ is a net contribution from enthalpic by nature intermolecular ligand-DNA interactions (H-bonds, electrostatic and van der Waals forces); $\Delta G_{\text {hyd }}$ is a hydrophobic contribution, computed from known empirical relation with the change in solvent accessible surface area, $\Delta A$ (SASA)

$\Delta G_{\text {hyd }}=0.00542 \cdot \Delta A / \mathrm{kcal} \cdot \mathrm{mol}^{-1}$

In case of intercalative binding $\Delta G_{\text {conf }}$ is mainly determined by formation of intercalation cavity in DNA, whereas the change of conformation of the ligand is considered negligible. The term $\Delta G_{\text {mol }}$ within the Chaires's approach is not computed as an independent contribution, and is calculated as a difference of $\Delta G_{\exp }$ and the sum of the rest terms in Eq. (2). This method has been successfully applied by numerous authors for energy analysis of the complexation reactions for intercalators (e.g. [45]) and MGBligands (e.g. [46-48]).

Although being computationally non-demanding and not requiring the use of molecular modeling techniques, the method by Chaires et al. does not provide the solution of the energy decomposition task, because the very important intermolecular term $\Delta G_{\mathrm{mol}}$ is not computed independently on $\Delta G_{\text {exp }}$, and does not allow further decomposition on electrostatic, van der Waals and hydrogen bonding terms. Moreover, for the group of ligand-DNA interactions the $\Delta G_{\mathrm{mol}}$ terms was shown to be relatively small resulting in conclusion that intermolecular forces just act as 'a fine tuning' of the binding energetics and are relatively unimportant. The full energy analysis is, thus, not possible within this approach.

Detailed exploration of Eq. (2) was accomplished by Baginski et al. with respect to energy analysis of DNA binding of a set of anthracycline antibiotics [49]. These authors additionally introduced explicit account of electrostatic interactions and vibrational contribution, although the van der Waals and H-bonding terms were not discussed based on the assumption that van der Waals and H-bonding terms compensate each other. It should be noted that the calculated sum of energy terms, $\Delta G_{\text {total }}$, had coincided with experimental energy, $\Delta G_{\text {exp }}$, in this work, although this result was achieved for the group of structurally similar anthracycline drugs.

\subsection{The method by Kollman et al.}

The approach developed by Kollman et al. (see for review [5053]), is based on the use of molecular dynamics simulation (MD) and continuum solvent model, and can be considered as a basic method of energy analysis of biomolecular binding interactions. It has, however, been applied mainly for protein binding reactions 


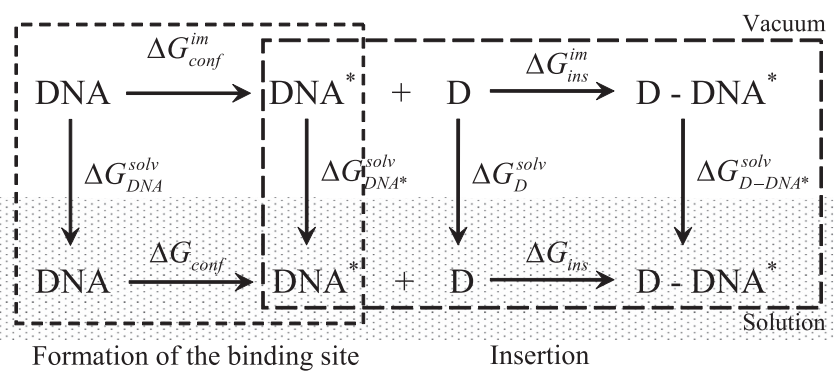

Fig. 1. Thermodynamic cycle for the ligand-NA binding process.

with very rare applications to ligand-NA interactions (e.g. $[52,54,55])$. Within the Kollman's approach the net Gibbs energy of complexation is computed as [50]

$\Delta G_{\text {total }}=\Delta E_{\mathrm{MM}}+\Delta G_{\mathrm{PBSA}}-T \Delta S_{\mathrm{MM}}$

where $\Delta E_{\text {мм }}$ is trajectory averaged MD energy, which includes deformation of chemical bonds, angles, intra- and intermolecular van der Waals and electrostatic terms; $-T \Delta S_{\text {мм }}$ is the net entropic contribution, calculated from normal mode analysis; $\Delta G_{\mathrm{PBSA}}=\Delta G_{\mathrm{PB}}+\Delta G_{\mathrm{np}}$ is a contribution from solvent, containing electrostatic solvation, $\Delta G_{\mathrm{PB}}$, and non-polar (van der Waals and hydrophobic), $\Delta G_{\mathrm{np}}$, energies. The $\Delta G_{\mathrm{PB}}$ terms is commonly calculated using Born approach, and $\Delta G_{\mathrm{np}}$ is calculated from empirical relation

$\Delta G_{\mathrm{np}}=(0.00542 \cdot \Delta A--0.92) / \mathrm{kcal} \cdot \mathrm{mol}^{-1}$

The Kollman's method was incorporated into the MMPBSA [solution of non-linear Poisson-Boltzmann equation (NLPB method) for electrostatics] and MMGBSA (Born method for electrostatics) modules of AMBER [56] and used for energy analyses of ligand binding with DNA [57-61] and RNA [62,63]. Although the Kollman's method contains the most detailed decomposition of the net Gibbs energy change onto energy terms and uses wellestablished procedures of molecular modelling, the successful decomposition of the net energy specifically for ligand-NA complexations was reported in relatively rare cases (e.g. $[12,60,63])$.

\subsection{The method by Evstigneev et al.}

The method suggested by Evstigneev et al. is essentially an extension of the Kollman's approach, specifically designed for energy analysis of ligand-NA complexation. It had resulted in good correspondence of between $\Delta G_{\text {total }}$ and $\Delta G_{\exp }$ for wide variety of DNA intercalation reactions $[26,64,65]$, minor groove binding [25], ligand binding with RNA [66] and parsing of stacking interactions in complexes of aromatic molecules [67]. The essence of this approach is grounded on the following considerations:

(i) binding of the ligand must be accompanied by formation of the binding site on NA, which is commonly referred to as DNA unwinding (i.e. transition of the DNA helix from a regular B-form into an unwound $D_{N A}^{*}$ ) for the intercalation process, and DNA or RNA adaptation - for DNA minor groove binding or ligand-RNA binding processes. Hence, the total energy of binding, $\Delta G_{\text {total }}$, should be decomposed onto two parts: the energy of NA conformational change, $\Delta G_{\text {conf }}$, and the energy of ligand insertion, $\Delta G_{\text {ins }}$

$\Delta G_{\text {total }}=\Delta G_{\text {conf }}+\Delta G_{\text {ins }}$.

(ii) NA-binding process occurs in solution, which means that the total Gibbs energy should be partitioned onto inter- or intramolecular interactions of NA and ligand in vacuum, $\Delta G^{\mathrm{im}}$, and their interaction with solvent, $\Delta G^{\text {solv }}$ :
$\Delta G_{\text {total }}=\Delta G^{\mathrm{im}}+\Delta G^{\mathrm{solv}}$

The dissection of the total energy on solvation/intermolecular (Eq. (7)) and on conformation/insertion (Eq. (6)) terms can be incorporated into a thermodynamic cycle (Fig. 1). The use of thermodynamic cycle suggests that at least two different ways for energy decomposition may exist:

(i) decomposition in terms of physical interactions - Eq. (8)

$$
\begin{aligned}
\Delta G_{\text {total }}= & \Delta G_{\text {conf }}+\Delta G_{\mathrm{vdW}}+\Delta G_{\mathrm{el}}+\Delta G_{\mathrm{pe}}+\Delta G_{\mathrm{hyd}}+\Delta G_{\mathrm{HB}} \\
& +\Delta G_{\mathrm{CT}}+\Delta G_{\mathrm{entr}}
\end{aligned}
$$

(ii) further decomposition of the 'vdW', 'el' and 'HB' terms in Eq. (8) in terms of the types of interaction (intermolecular interactions in vacuum and with solvent) - Eq. (9)

$$
\begin{aligned}
\Delta G_{\text {total }}= & \Delta G_{\text {conf }}+\Delta G_{\mathrm{vdW}}^{\mathrm{im}}+\Delta G_{\mathrm{vdW}}^{\text {solv }}+\Delta G_{\mathrm{el}}^{\mathrm{im}}+\Delta G_{\mathrm{el}}^{\mathrm{solv}}+\Delta G_{\mathrm{pe}} \\
& +\Delta G_{\mathrm{hyd}}+\Delta G_{\mathrm{HB}}^{\mathrm{im}}+\Delta G_{\mathrm{HB}}^{\mathrm{solv}}+\Delta G_{\mathrm{CT}}+\Delta G_{\mathrm{entr}}
\end{aligned}
$$

where $\Delta G_{\mathrm{vdW}}$ and $\Delta G_{\mathrm{el}}$ are the contributions from van der Waals (vdW) and electrostatic (el) interactions, respectively, $\Delta G_{\mathrm{pe}}$ is the polyelectrolyte (PE) contribution, $\Delta G_{\text {hyd }}$ is the hydrophobic (hyd) contribution, $\Delta G_{\mathrm{HB}}$ is the contribution from hydrogen bonds (HB), $\Delta G_{\mathrm{CT}}$ is the charge-transfer contribution (CT), and $\Delta G_{\text {entr }}$ is the entropic term originating from changes in the net number of degrees of freedom of the investigated system.

The entropic term, $\Delta G_{\text {entr, }}$, originates from the loss of translational $\left(\Delta G_{\mathrm{t}}\right)$, rotational $\left(\Delta G_{\mathrm{r}}\right)$ degrees of freedom, change in the mode of vibrations of chemical bonds (the high frequency term or type I vibrations, $\Delta G_{v}^{\mathrm{l}}$, ) and appearance of new mechanical oscillations of the ligand in the binding site (the low frequency term or type II vibrations $\Delta G_{v}^{\mathrm{II}}$ ), i.e.

$\Delta G_{\mathrm{entr}}=\Delta G_{\mathrm{t}}+\Delta G_{\mathrm{r}}+\Delta G_{\mathrm{v}}^{\mathrm{I}}+\Delta G_{\mathrm{v}}^{\mathrm{II}}$

The computation of each of the terms in Eqs. (8) and (9) generally follows the Kollman et al. approach, viz. the use of continuum solvent model for electrostatics within the framework of NLPB approach, the use of free energy perturbation for the formation of binding site, averaging of van der Waals energy during MD and normal mode analysis of vibrational contribution. There are three principal differences between the Kollman et al. and the Evstigneev et al. approaches:

(i) the decomposition of non-polar contribution, $\Delta G_{\mathrm{np}}$, onto 'hyd' and 'vdW' with solvent: $\Delta G_{\mathrm{np}}=\Delta G_{\mathrm{hyd}}+\Delta G_{\mathrm{vdW}}^{\text {solv }}$ with explicit computation of the VDW part in MD and HYD contribution as

$\Delta \mathrm{G}_{\mathrm{hyd}}=0.05 \cdot \Delta \mathrm{A} / \mathrm{kcal} \cdot \mathrm{mol}^{-1}$

(ii) introduction of low frequency mechanical oscillations of the ligand in binding site, $\Delta G_{\mathrm{v}}^{\mathrm{II}}$, partially restoring the loss of translational and rotational degrees of freedom, and

(iii) explicit account for the energy of change in the number of intermolecular $\mathrm{HB}\left(\Delta G_{\mathrm{HB}}^{\mathrm{im}}\right)$ and $\mathrm{HB}$ with solvent $\left(\Delta G_{\mathrm{HB}}^{\mathrm{solv}}\right)$.

A special note should be given to the method of explicit account of the energy of $\mathrm{H}$-bonds, $\Delta G_{\mathrm{HB}}$. Within the framework of the Evstigneev et al. approach it is considered that part of the $\mathrm{H}$ bond energy is already accounted for in the $\Delta G_{\mathrm{vdw}}$ and $\Delta G_{\mathrm{el}}$ terms, hence, the $\Delta G_{\mathrm{HB}}$ quantity bears meaning of an additional amount to the sum of van der Waals and electrostatic energies in order 
to account correctly for the total contribution due to H-bonding. The energy contribution from hydrogen bonds to water molecules on complexation and intermolecular $\mathrm{H}$-bonds can be estimated from the change in hydration index of the system (i.e. $N^{\mathrm{im}}$ or $\Delta N^{\text {solv }}$ representing the average number of intermolecular $\mathrm{H}$-bonds and change in the number of $\mathrm{H}$-bonds to water molecules on complexation, respectively) and further calculation of $\Delta G_{\mathrm{HB}}$ by means of the formula

$\Delta G_{\mathrm{HB}}=-0.25 \cdot 9 \cdot\left(N^{\mathrm{im}}+\Delta N^{\mathrm{solv}}\right) / \mathrm{kcal} \cdot \mathrm{mol}^{-1}$

Below we shall briefly discuss the results and main outcomes of the energy analyses of the ligand-NA complexation reactions.

\section{The contribution from van der Waals energy}

Table 1 contains the dispersion range of various terms of van der Waals energy for various types of ligand-NA complexations, collected from the results reported by different research groups. The following general patterns of van der Waals energy have been observed.

The intramolecular energies in NA, $\Delta G_{\text {conf }}^{\mathrm{im}}$, at the stage of binding site formation are mainly positive, which is a result of perturbation of NA structure on ligand binding (e.g. separation of base pairs upon formation of the intercalation cavity). The energies of the solvation of the binding site, $\Delta G_{\text {conf }}^{\text {solv }}$, are mainly negative and result from additional hydration of the binding site upon NA perturbation on complexation. For DNA intercalation the $\Delta G_{\text {conf }}$ term is much higher than that for DNA minor-groove and RNA bindings which is quite expected due to most significant changes of DNA structure required for the formation of intercalation cavity. Interestingly, no apparent correlation of the van der Waals terms with the unwinding angle was observed [26], although this correlation is the case for electrostatic energy (see below).

The average values of $\Delta G_{\text {conf }}$ (the adaptation energy) for MGBand RNA-bindings relatively small and not simple to rationalize in terms of structure of the binding site or the ligand, partially because different authors use different approaches to estimate this term. As a consequence, there is some controversy in literature regarding its contribution to binding energetics. Any structural changes in either the DNA or the ligand for non-covalent binding with DNA groove are often assumed to be negligible or distributed over other energy terms $[25,44,46-48,68,69]$. The ligand itself provides one more contribution to the $\Delta G_{\text {conf }}$ term originating from restriction of internal rotations in the molecule (especially for MGB-ligands), thus lowering the total number of conformations of the ligand allowed within the complex as compared to the free ligand in solution (this term, however, is considered to be small as compared with vibrational contribution $[25,70]$. From the other hand, in other approaches the calculated energy of conformational change of MGB-DNA complex may reach $50.2 \mathrm{~kJ} \cdot \mathrm{mol}^{-1}$ $\left(12 \mathrm{kcal} \cdot \mathrm{mol}^{-1}\right)$ [13], which is a significant contribution to the total Gibbs energy change. Unfortunately, for RNA binding the formation of some general view on $\Delta G_{\text {conf }}$ term is still not possible due to very limited data available. The high conformational flexibility of the binding site in RNA aptamer creates certain difficulties in reliable modelling of the $\Delta G_{\text {conf }}$ term discussed in more detail in $[54,66,71,72]$.

At the stage of ligand insertion the intermolecular energy of ligand-NA interaction, $\Delta G_{\text {ins }}^{\text {im }}$, has a negative sign, which results from the attractive nature of vdW forces acting between the ligand and NA base pairs within the binding site. According to quantummechanical calculations this term is dominated by London dispersive forces [73,74] and demonstrates correlation with aromatic area of the ligand chromophore in case of DNA intercalation [64], some instances of RNA binding and other aromatic-aromatic interactions [75-77]. The disruption of stacking is observed for some RNA binding ligands and results in lowering the $\Delta G_{\text {ins }}^{\text {im }}$ term by absolute value (e.g. [54]). Noteworthy, the $\Delta G_{\text {ins }}^{\text {im }}$ term was also reported to be sensitive to structural complementarity of the ligand and the base-pairs, as demonstrated by elliptic binding with DNA [64] and malachite green binding with RNA [55,78].

The positive VDW energy of the interaction with solvent, $\Delta G_{\text {ins }}^{\text {solv }}$, is due to dehydration of the ligand after its insertion into NA interior. The van der Waals solvation energy was reported to qualitatively correlate with the mass and bulkiness of side chains of the ligand and/or with the change in SASA for all the groups of ligand-NA interactions discussed here [13,79]. The values of $\Delta G_{\text {ins }}^{\text {im }}$ and $\Delta G_{\text {ins }}^{\text {solv }}$ terms are, on average, few-fold higher than the experimental energy of binding ( $\left.\mathrm{ca} .-42 \mathrm{~kJ} \cdot \mathrm{mol}^{-1}\left(-10 \mathrm{kcal} \cdot \mathrm{mol}^{-1}\right)\right)$ $[26,49,64]$.

The total van der Waals energy of insertion, $\Delta G_{\text {ins }}$, is a relatively small value, commensurate with $\Delta G_{\text {exp }}$, and is a result of mutual compensation from favourable intermolecular interaction between NA and the ligand, and unfavourable interaction with the solvent (see Table 1). The compensation may lead to positive and negative $\Delta G_{\text {ins }}$ which means that van der Waals interactions at the stage of insertion may either favour or disfavour complex formation as it depends on the interplay between the intermolecular interactions and the interactions with solvent.

The total van der Waals energy of binding, $\Delta G_{\mathrm{vdW}}$, is the sum of two large numbers, $\Delta G_{\mathrm{vdW}}^{\mathrm{im}}+\Delta G_{\mathrm{vdW}}^{\mathrm{solv}}$, of opposite sign, which results in a small net energy effect for DNA intercalation and MGB binding. The most prominent feature of $\Delta G_{\mathrm{vdw}}$ term is the fact that it does not demonstrate systematic correlation with structural properties of ligand or NA $[25,26]$, whereas this correlation is observed on the level of the components of van der Waals energy (such as the correlation with the area or mass of the ligand with 'im' component and the bulkiness of side chains with 'solv' component,

Table 1

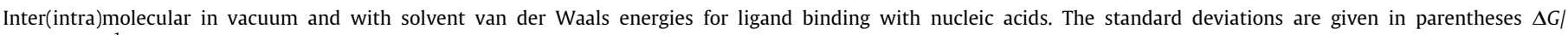
$4.18 \mathrm{~kJ} \cdot \mathrm{mol}^{-1}$.

\begin{tabular}{|c|c|c|c|c|c|c|c|c|c|c|}
\hline \multirow[t]{2}{*}{ Type of complexation } & \multicolumn{3}{|c|}{ Formation of the binding site } & \multicolumn{3}{|c|}{ Insertion to the binding site } & \multicolumn{3}{|c|}{ Overall binding } & \multirow[t]{2}{*}{ Refs. } \\
\hline & $\Delta G_{\mathrm{conf}}^{\mathrm{im}}$ & $\Delta G_{\text {conf }}^{\text {solv }}$ & $\Delta G_{\text {conf }}$ & $\Delta G_{\text {ins }}^{\text {im }}$ & $\Delta G_{\text {ins }}^{\text {solv }}$ & $\Delta G_{\text {ins }}$ & $\Delta G_{\mathrm{vdW}}^{\mathrm{im}}$ & $\Delta G_{\mathrm{vdW}}^{\mathrm{solv}}$ & $\Delta G_{\mathrm{vdW}}$ & \\
\hline DNA intercalation & $\begin{array}{l}28.5 \\
(6.6)\end{array}$ & $\begin{array}{l}-16.3 \\
(8.5)\end{array}$ & $\begin{array}{l}12.2 \\
(8.2)\end{array}$ & $\begin{array}{l}-61.9 \\
(20.5)\end{array}$ & $\begin{array}{l}53.3 \\
(29.1)\end{array}$ & $\begin{array}{l}8.6 \\
(12.9)\end{array}$ & $\begin{array}{l}-33.4 \\
(20.4)\end{array}$ & $\begin{array}{l}37.0 \\
(25.4)\end{array}$ & $\begin{array}{l}3.6 \\
(9.5)\end{array}$ & {$[15,26,49,57]$} \\
\hline DNA minor-groove binding & $-1)$ & & $\sim 1^{2)}$ & $\begin{array}{l}-57.6 \\
(7.7)\end{array}$ & $\begin{array}{l}51.5 \\
(11.2)\end{array}$ & $\begin{array}{l}-6.1 \\
(7.7)\end{array}$ & $\begin{array}{l}-57.6 \\
(7.7)\end{array}$ & $\begin{array}{l}51.5 \\
(11.2)\end{array}$ & $\begin{array}{l}-6.1 \\
(7.7)\end{array}$ & {$[13,15,25]$} \\
\hline RNA aptamer complexation & $-{ }^{1)}$ & & & $\begin{array}{l}-50.5 \\
(12.1)\end{array}$ & $\begin{array}{l}31.5 \\
(7.4)\end{array}$ & $\begin{array}{l}-19.0 \\
(9.3)\end{array}$ & $\begin{array}{l}-50.5 \\
(12.1)\end{array}$ & $\begin{array}{l}31.5 \\
(7.4)\end{array}$ & $\begin{array}{l}-19.0 \\
(9.3)\end{array}$ & {$[54,66]$} \\
\hline
\end{tabular}

1) The value of energy the terms is not possible to estimate from available literature data.

2) The value of the energy term is small. 
mentioned above). It is, probably, the consequence of different by weight and usually uncorrelated contribution of the 'chromophore' and 'side chains' of the ligand to the 'im' and 'solv' components of the van der Waals energy.

It is worth noting that the overall balance of the 'im' and 'solv' van der Waals energies in case of RNA binding is shifted towards the 'im' term (see Table 1), enabling to conclude that intermolecular van der Waals interactions appear to be most pronounced specifically in RNA binding reactions. In contrast to this, the 'im' and 'solv' terms, on average, counterbalance each other, leading to quite natural conclusion that van der Waals interactions do not play a significant role in ligand-NA binding. This is correct in terms of overall binding but not correct in terms of stabilization of the complexes. The portion of the total van der Waals energy of ligand binding, $\Delta G_{\text {ins }}$, discussed above, which really contributes to stabilization of the complex, may take the values (see Table 1) higher by modulus than the experimentally-measured energies of binding, $\Delta G_{\exp }[21,25,26,80-82]$. Hence, it is always necessary to take into account the contributions of VDW interactions at different stages of binding (site formation and insertion) and for different types of interaction (intermolecular and with solvent) for correct energy analysis of ligand-NA complexations.

One final note regarding the computation of van der Waals terms is worth mentioning. The van der Waals solvation component of the net energy of binding is one of the most difficult in terms of computation as it is strongly dependent on structure, parameterization and evolution time used in MD simulation, and sometimes causes discrepancies of the results of computations reported by different authors $[13,25]$. Another difficulty is related to intrinsically small values of the net van der Waals energy, $\Delta G_{\mathrm{vdW}}$, obtained as a difference of large numbers. As a consequence, the error in computation of $\Delta G_{\mathrm{vdW}}$ using modern force fields for the group of NA binding ligands may commensurate with $\Delta G_{\mathrm{vdW}}$ itself, making this quantity unreliable in energy analysis [25-27]. Partial way out, commonly used in some packages of molecular modelling (e.g. in AMBER) and wide-spread in ligandNA energy analysis (e.g. $[58,59,83,84])$, is incorporation of empirical interrelation between change in SASA and $\Delta G_{\mathrm{vdW}}^{\text {solv }}$ (sometimes referred to as non-polar term, see also Eq. (5)), viz. $\Delta G_{\mathrm{vdW}}^{\mathrm{solv}}=\gamma_{v d W} \cdot \Delta A$ (where $\gamma_{\mathrm{vdW}}$ takes the values $-(125 \ldots 167)$ $\mathrm{J} \cdot \mathrm{mol}^{-1} \cdot \AA^{-2}$ or $-(21 \ldots 29) \mathrm{J} \cdot \mathrm{mol}^{-1} \cdot \AA^{-2}$ or $\{-(30 \ldots 40)$ or $(5 \ldots 7)$ $\left.\mathrm{cal} \cdot \mathrm{mol}^{-1} \cdot \AA^{-2}\right\}$ depending on whether the hydrophobic interaction is included or not during evaluation of the solvation energy), initially derived experimentally for simple hydrocarbons [85]. It has, however, been shown [79] that specifically for ligand-NA complexations the exact value of the $\gamma_{\mathrm{vdw}}$ coefficient may remarkably vary with the type of interaction studied, and may partly account for the above-mentioned fact that the solution of energy decomposition problem for ligand-NA interactions has been much less extensively reported in literature, as compared to proteinbinding systems.

\section{The contribution from electrostatic energy}

The dispersion range of values of various terms for the electrostatic energy of the ligand binding to NA is given in Table 2.

It is seen from Table 2 that specifically for DNA intercalation the change in the electrostatic component of the energy of interaction with the surrounding water, $\Delta G_{\text {conf }}^{\text {solv }}$, upon DNA unwinding is positive. These observations were explained in terms of the decrease in charge density on the DNA surface as a result of unwinding, which inevitably causes the weakening of interaction with water surrounding $[49,86]$. The contribution of intramolecular interactions to the Gibbs energy of unwinding, $\Delta G_{\mathrm{conf}}^{\mathrm{im}}$, is negative, i.e. this type of interaction promotes the unwinding of DNA molecule. This behaviour results from the increase in distance between the negatively-charged phosphates on formation of the intercalation cavity, which as a whole is an energetically favourable process $[49,86]$. A good correlation between the values of the untwist angle, $\Delta \Omega$, of the DNA duplex upon intercalation of the ligands and the calculated energy, $\Delta G_{\text {conf }}^{\text {im }}$, of intramolecular coulomb interactions inside the double helix on unwinding was reported [86]: the greater the $\Delta \Omega$ angle, the greater are the negative changes of $\Delta G_{\text {conf }}^{\mathrm{im}}$. This conclusion is supported, in part, by a known correlation between the electrostatic potential in the centre of the intercalation site with an increase of $\Omega$ angle [87]. Importantly, both electrostatic terms, $\Delta G_{\mathrm{conf}}^{\mathrm{im}}$ and $\Delta G_{\mathrm{conf}}^{\text {solv }}$, have been shown to depend strongly on the structure of the intercalation site, and, eventually, on the type of ligand studied [86], which makes it necessary to account for these terms explicitly in energy analysis.

The total DNA unwinding electrostatic energy, $\Delta G_{\text {conf }}$, is relatively small value with alternating sign depending on ligand, and is formed as a sum of large numbers having opposite signs (see Table 2). No clear correlation of its value with the structure of intercalation site was reported $[49,86]$.

In contrast to conformational electrostatic energy, the electrostatic terms related to ligand insertion, $\Delta G_{\text {ins }}^{\text {im }}$ and $\Delta G_{\text {el }}^{\text {im }}$, exert clear correlation with either, the sign of the ligand charge, and change in SASA. The change in the energies of intermolecular interaction is negative for positively-charged ligands, and small and positive for neutral ligands. Positively-charged ligands provide energetically favourable electrostatic interaction with negatively-charged phosphates in DNA and RNA which, in turn, leads to large-byabsolute-value negative values of $\Delta G_{\text {ins }}^{\mathrm{im}}$ and $\Delta G_{\mathrm{el}}^{\mathrm{im}}$. For the neutral molecules the sign and value of the components of the 'im' electrostatic energies are determined by immediate distribution of charge on the molecule.

The solvation energies, $\Delta G_{\text {ins }}^{\text {solv }}$ and $\Delta G_{\text {el }}^{\text {solv }}$, may either be positive and negative which is a result of the ligand and DNA/RNA desolvation on complex formation (see Table 2). Both components have been reported to correlate with change in SASA and the sign of

Table 2

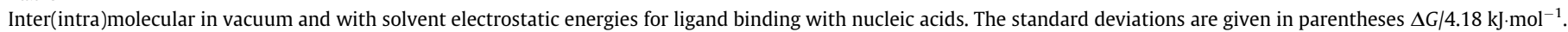

\begin{tabular}{|c|c|c|c|c|c|c|c|c|c|c|}
\hline \multirow[t]{2}{*}{ Type of complexation } & \multicolumn{3}{|c|}{ Formation of the binding site } & \multicolumn{3}{|c|}{ Insertion to the binding site } & \multicolumn{3}{|c|}{ Overall binding } & \multirow[t]{2}{*}{ Refs. } \\
\hline & $\Delta G_{\mathrm{conf}}^{\mathrm{im}}$ & $\Delta G_{\text {conf }}^{\text {solv }}$ & $\Delta G_{\text {conf }}$ & $\Delta G_{\text {ins }}^{\text {im }}$ & $\Delta G_{\text {ins }}^{\text {solv }}$ & $\Delta G_{\text {ins }}$ & $\Delta G_{\mathrm{el}}^{\mathrm{im}}$ & $\Delta G_{\mathrm{el}}^{\text {solv }}$ & $\Delta G_{\mathrm{el}}$ & \\
\hline DNA intercalation & $\begin{array}{l}-29.1 \\
(9.8)\end{array}$ & $\begin{array}{l}28.5 \\
(12.4)\end{array}$ & $\begin{array}{l}-0.6 \\
(5.6)\end{array}$ & $\begin{array}{l}-92.3 \\
(75.9)\end{array}$ & $\begin{array}{l}93.4 \\
(71.2)\end{array}$ & $\begin{array}{l}1.1 \\
(6.4)\end{array}$ & $\begin{array}{l}-121.4 \\
(68.3)\end{array}$ & $\begin{array}{l}121.9 \\
(62.7)\end{array}$ & $\begin{array}{l}0.5 \\
(9.9)\end{array}$ & {$[49,86,87]$} \\
\hline DNA minor-groove binding & $-1)$ & & $\sim 1^{2)}$ & $\begin{array}{l}-233.6 \\
(54.3)\end{array}$ & $\begin{array}{l}235.6 \\
(54.6)\end{array}$ & $\begin{array}{l}2.0 \\
(2.1)\end{array}$ & $\begin{array}{l}-233.6 \\
(54.3)\end{array}$ & $\begin{array}{l}235.6 \\
(54.6)\end{array}$ & $\begin{array}{l}2.0 \\
(2.1)\end{array}$ & {$[25]$} \\
\hline RNA aptamer complexation & $-{ }^{1)}$ & & & $\begin{array}{l}-134.0 \\
(232.1)\end{array}$ & $\begin{array}{l}129.3 \\
(220.8)\end{array}$ & $\begin{array}{l}-4.7 \\
(11.5)\end{array}$ & $\begin{array}{l}-134.0 \\
(232.1)\end{array}$ & $\begin{array}{l}129.3 \\
(220.8)\end{array}$ & $\begin{array}{l}-4.7 \\
(11.5)\end{array}$ & [66] \\
\hline
\end{tabular}

Notes:

1) The value of the energy terms is not possible to estimate from available literature data.

2) The value of the energy term is small. 
the ligand $[25,49,66,86]$. When the positively-charged molecules bind with DNA or RNA, their charges are compensated by the negative charge of the neighbouring DNA/RNA phosphates, which leads to an overall weakening of electrostatic interaction with solution and small positive or negative values of the 'solv' components. If the ligand is negatively-charged, the complexation results in increase of linear charge density of DNA/RNA and favourable interaction with solvent [86]. The electrostatic energy has been reported to be of greatest importance specifically for the group of MGB-ligands [25] and RNA-binders [66]. The dispersion of electrostatic 'im' and 'solv' terms for RNA-binders by sign and value appears to be the highest within the group of small molecules studied in the cited works as compared with DNA intercalative and minor-groove bindings.

Importantly, the size of the $\Delta G_{\mathrm{el}}^{\text {solv }}$ and $\Delta G_{\mathrm{el}}^{\mathrm{im}}$ terms are nearly equal by absolute value resulting after summation in a small number for the total electrostatic energy of the complexation reaction, $\Delta G_{\mathrm{el}}$, for all groups of interactions discussed here (see Table 2). The $\Delta G_{\text {el }}$ quantity does not feature any apparent correlation to the type or charge of the ligands $[25,26,64,66,86]$. As already discussed above for the van der Walls energy, this result is quite natural since the error in the computation of $\Delta G_{\mathrm{el}}^{\text {solv }}$ and $\Delta G_{\mathrm{el}}^{\mathrm{im}}$ terms commensurate with $\Delta G_{\mathrm{el}}$ itself, which in fact makes an analysis of the total electrostatic energy meaningless. Hence, it is considered that any search for a correlation between the structure of a ligand and its energy of complexation should only be made at the level of separate stages of the complexation process and appropriate components of the electrostatic energy rather than in terms of total electrostatic energy.

Some of the patterns described above have been noted above for the group of aromatic DNA intercalators, which presumably reflects the general trends associated with the electrostatic energy change occurring on non-covalent ligand-NA binding reactions in aqueous solution [88] (to be discussed below).

One note regarding the computation of electrostatic energy terms is worth mentioning. The magnitude of this type of interaction might be strongly dependent on the type of force field employed, i.e. the method of electric charge computation on the ligand and DNA/ RNA receptor. Although the charge distribution and magnitudes of charges have actually been shown to depend on the underlying method of calculation, the absolute values of electrostatic energies of ligand-NA interactions have been reported to depend to much lesser extent on the computation scheme employed [89], in many cases enabling to perform comparative analysis of the results from different research groups. The method used for electrostatic energy evaluation is also important as it should correctly handle such effect as polarization of water environment and the reactants. To the best of our knowledge in issues related to energy analysis and energy decomposition, the NLPB and Born's electrostatic models of continuum solvent were reported to perform well.

\section{The contribution from hydrophobic energy}

Prior to analysis of the contribution from hydrophobic interactions, a special discussion should be given to the method of their calculation.
Currently in analyses of hydrophobic contribution to the binding reactions in solution the major utilization has received two main empirical approaches. In series of works undertaken by Chaires's group [19,42-44] and some other authors (e.g. Refs. $[47,48]$ ) in energy analyses of DNA intercalation and minor groove binding reactions the following empirical relation for the energy of hydrophobic contribution was used, measured previously for folding of proteins at $T=298 \mathrm{~K}[90-92]$ (the ' $\Delta C_{\mathrm{p}}$-method')

$\Delta G_{\text {hyd }}=80 \cdot \Delta C_{\mathrm{p}} / \mathrm{cal} \cdot \mathrm{mol}^{-1}$,

where $\Delta C_{\mathrm{p}}$ is given in cal $\cdot \mathrm{K}^{-1} \cdot \mathrm{mol}^{-1}$.

In its turn, $\Delta C_{\mathrm{p}}$ linearly correlates with the change in non-polar SASA, $\Delta A_{\mathrm{np}}$ [91] or, in more recent work [92] of the same authors, with both the change in polar $\Delta A_{\mathrm{p}}$ and non-polar $\Delta A_{\mathrm{np}}$ surface areas SASA of protein-nucleic acid complexes. In Ref. [42] this linear correlation of $\Delta G_{\text {hyd }}$ with $\Delta A_{n p}$ and $\Delta A_{\mathrm{p}}$ was adjusted from fitting of a wide set of calorimetric data for ligand-DNA to the form

$\Delta \mathrm{C}_{\mathrm{p}}=0.382 \cdot \Delta \mathrm{A}_{\mathrm{np}}-0.121 \cdot \Delta \mathrm{A}_{\mathrm{p}} / \mathrm{cal} \cdot \mathrm{K}^{-1} \mathrm{~mol}^{-1}$

where $\Delta A$ is given in $\AA^{2}$.

In the cited works, the values of the hydrophobic contribution was found to fall in between $\Delta G_{\mathrm{hyd}} \approx-(46 \ldots 54) \mathrm{kJ} \cdot \mathrm{mol}^{-1}$ or $\left\{-(11 \ldots 13) \mathrm{kcal} \cdot \mathrm{mol}^{-1}\right\}$ for intercalators, and $\Delta G_{\mathrm{hyd}} \approx-(63-105)$ $\mathrm{kJ} \cdot \mathrm{mol}^{-1}$ or $\left\{-(15 \ldots 25) \mathrm{kcal} \cdot \mathrm{mol}^{-1}\right\}$ for MGB-ligands. Hence the hydrophobic interactions were reported to stabilize the ligandDNA complexes to significant extent, and specifically for MGBligands the hydrophobic contribution is higher by absolute value than that for intercalators.

An alternative to Eq. (14) is the ' $\gamma$-method', based on the existence of experimentally verified correlation between the energy of hydrophobic dissolution of simple hydrocarbons and aminoacids $[49,87,93,94]$ and the change in SASA:

$\Delta \mathrm{G}_{\mathrm{hyd}}=\gamma \Delta \mathrm{A}$,

where $\gamma$ is microscopic surface tension coefficient, usually taken by many researches equal to $\gamma \approx 50 \mathrm{cal} \cdot \mathrm{mol}^{-1} \cdot \AA^{-2}$.

With this value of $\gamma$ Eq. (15) is being transformed into Eq. (11). This method was used to calculate the hydrophobic contribution to binding energetics of various intercalators [26,49,64,65], MGB[25,59] and RNA-binding [66] ligands (Table 3).

Qualitatively the results from the ' $\Delta C_{\mathrm{p}}$-method' and the ' $\gamma$ method' were reported to match each other, however, quantitatively the ' $\Delta C_{\mathrm{p}}$-method' have been shown to give underestimated values of $\Delta G_{\text {hyd }}$. For instance, the complexation of daunomycin and its derivatives with DNA is characterized by $\Delta G_{\text {hyd }}=-$ (146. . 201) $\mathrm{kJ} \cdot \mathrm{mol}^{-1}\left\{-(35 \ldots 48) \mathrm{kcal} \cdot \mathrm{mol}^{-1}\right\}$ from the ' $\gamma$-method', which is notably different from $\Delta G_{\mathrm{hyd}} \approx 53.5 \mathrm{~kJ} \cdot \mathrm{mol}^{-1}$ $\left(-12.8 \mathrm{kcal} \cdot \mathrm{mol}^{-1}\right)$, computed using the ' $\Delta C_{\mathrm{p}}$-method' in Ref. [42]. The origin of this effect has never been systematically investigated except the sole works $[26,95]$ reporting that the ' $\gamma$-method' is more appropriate than the ' $\Delta C_{\mathrm{p}}$-method' specifically for ligand-DNA interactions. Nevertheless, the results of both methods enable to provide a general view on the involvement of hydrophobic interactions into ligand-NA binding energetics.

Either for DNA intercalators and MGB-ligands, and RNAbinders, the sequence of ligands placed in the order by decrease

Table 3

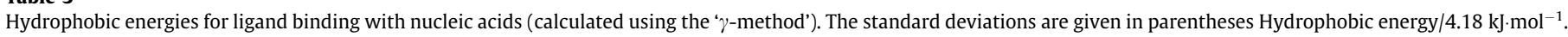

\begin{tabular}{|c|c|c|c|c|}
\hline Type of complexation & Formation of the binding site & Insertion to the binding site & Overall binding & Refs. \\
\hline DNA intercalation & $\begin{array}{l}10.7 \\
(2.9)\end{array}$ & $\begin{array}{l}-36.1 \\
(11.9)\end{array}$ & $\begin{array}{l}-25.4 \\
(10.1)\end{array}$ & {$[26,42,49]$} \\
\hline DNA minor-groove binding & $\sim 1$ & $\begin{array}{l}-40.9 \\
(7.1)\end{array}$ & $\begin{array}{l}-40.9 \\
(7.1)\end{array}$ & {$[25,44,46,48]$} \\
\hline RNA aptamer complexation & $\sim 1$ & $\begin{array}{l}-31.4 \\
(8.5)\end{array}$ & $\begin{array}{l}-31.4 \\
(8.5)\end{array}$ & [66] \\
\hline
\end{tabular}


of hydrophobic contribution, typically exerts apparent correlation with the degree of branching of the side chains of the investigated ligands, i.e. from large side chains of predominantly hydrophobic content down to ligands containing small hydrophilic groups. The bulkiness of the side chains influences the effectiveness of removal of water molecules from the hydration layers of DNA/ RNA and the ligand on complexation, also confirmed by the fact that the total change in solvent accessible surface area for the binding reactions is always negative. The hydrophobic contribution is thus, always favorable for either intercalation, MGB- and RNAbindings.

\section{The contribution from hydrogen bonds}

The contribution from hydrogen bonds to the net energy of ligand binding with NA is probably the most difficult for estimation because of quantum-chemical character of this interaction, strong dependence of the H-bond energy on the structure of the complex, need to make structural averaging during thermal motion, and the difficulty to separate the $\Delta G_{\mathrm{HB}}$ term from van der Waals and electrostatic energies. The quite extensive investigations of the $\mathrm{H}$ bonding energy in proteins or their components based on either experiment (e.g. [96-98]) or quantum-chemical calculations (e.g. $[99,100])$, as well as the relatively well characterized to date intramolecular H-bonding in nucleic acid bases (e.g. [101-103]), have, nevertheless, not been fully transferred to ligand-NA complexations, remaining this issue yet to be addressed.

Hydrogen bonding results from two sources in the complexation of ligands with NA:

(i) formation of intermolecular $\mathrm{H}$-bonds between the ligand and NA within the binding site, and

(ii) the loss (or formation) of hydrogen bonds-to-water due to dehydration of the ligand upon insertion into the binding site.

The topology and geometry of type (i) H-bonds in ligand-NA complexes (see for review [8]) have so far been extensively investigated. The total number of $\mathrm{H}$-bonds, $N_{\mathrm{im}}=(1 \ldots 5)$ for typical intercalators, $N_{\mathrm{im}}=(1 \ldots 11)$ for MGB-ligands and $N_{\mathrm{im}}=(0 \ldots 8)$ for RNA-binders, have been observed nearly for all ligand-NA complexes studied, although the energetic contribution to the net energy still remains unknown. It is a common practice to include the energy of H-bonding into the methods of molecular modelling as specially-adjusted potentials [104], although the explicit separation of the $\Delta G_{\mathrm{HB}}$ term has not been accomplished, except that of very few works (e.g. [105], where the H-bond energy of binding of berenil and pentamidine with DNA was estimated from molecular modelling, $\Delta G_{\mathrm{HB}} \approx-33 \mathrm{~kJ} \cdot \mathrm{mol}^{-1}\left(-8 \mathrm{kcal} \cdot \mathrm{mol}^{-1}\right)$. Some authors neglect all the energy contribution from $\mathrm{H}$-bonding, assuming that partial loss of $\mathrm{H}$-bonds-to-water (de-hydration) compensates the formation of intermolecular $\mathrm{H}$-bonds in such a way to always counterbalance the net effect down to zero (e.g. $[19,42,43,49])$. However, extensive investigations of hydration of hydrocarbons [96] have shown that the intermolecular and towater H-bonds differ from each other. The method of computation of the excess enthalpy of intermolecular $\mathrm{H}$-bond with respect to the H-bond to-water was developed based on shifts of resonance absorption peaks in IR spectra, $\Delta H=-(5.9 \ldots 7.5) \mathrm{kJ} \cdot \mathrm{mol}^{-1}$ $\left\{-(1.4 \ldots 1.8) \mathrm{kcal} \cdot \mathrm{mol}^{-1}\right\}$ [106]. It is thought that the difference between the intermolecular $\mathrm{H}$-bond and the $\mathrm{H}$-bond-to-water is a significant negative entropic contribution to the Gibbs energy of the latter originating from loss of translational and rotational freedom of water molecules captured by the bond [96,107], hence, the average Gibbs energy of H-bonding to-water is lower by modulus than the enthalpy of intermolecular $\mathrm{H}$-bond in low dielectric medium. In summary, it may be concluded that in the context of solving the energy decomposition problem the energy of intermolecular H-bonds should not be neglected for ligand binding with nucleic acids in aqueous solution.

A systematic investigation of the energy effect of $\mathrm{H}$-bonding for ligand-NA interactions was accomplished in a series of works $[25,26,64,108]$ based on empirical methodology described in Section 4.3, and allowing to estimate $\Delta G_{\mathrm{HB}}$ as an additional amount to the sum of van der Waals and electrostatic energies in order to account correctly for the total contribution due to H-bonding. The key result is that the net loss of $\mathrm{H}$-bonds to-water (i.e. dehydration) of the ligand and NA on complexation overbalances the formation of $\mathrm{H}$-bonds leading to the net energetically unfavourable (or nearly zero) contribution of the $\Delta G_{\mathrm{HB}}$ term to $\Delta G_{\text {total }}$ The overall de-hydration of the ligand and NA were found to correlate with the loss of solvent accessible surface area, $\Delta A$, and the value of the hydrophobic effect in reaction of binding.

\section{The polyelectrolyte contribution}

The polyelectrolyte contribution contains both an enthalpic term, originating from coulomb interaction of solute molecule with counterions present in solution, and an entropic term, coming from disordering of ion atmosphere upon ligand intercalation. In majority of works $\Delta G_{\mathrm{pe}}$ component is separated from the total electrostatic energy because it can be measured experimentally. The common approach to get $\Delta G_{\mathrm{pe}}$ is to measure dependence of equilibrium ligand-NA complexation constant on salt concentration (usually $\mathrm{Na}^{+}$). The data on various NA-binding ligands are available in literature for DNA (e.g. [6]) and RNA-binders (e.g. [109,110]). It is also commonly assumed that the main contribution to the polyelectrolyte energy comes from the ligand insertion stage, i.e. $\Delta G_{\text {conf }} \approx 0$. It was found that under physiological salt concentration $(\sim 0.1 \mathrm{M})$ the value of $\Delta G_{\mathrm{pe}}$ is $c a$. (4..13) $\mathrm{kJ} \cdot \mathrm{mol}^{-1}\{-(1 \ldots 3)$ $\left.\mathrm{kcal} \cdot \mathrm{mol}^{-1}\right\}$, weakly depends on the type of ligand and favours the complexation with NA. It appears that the polyelectrolyte contribution is the sole term of the net Gibbs energy which is relatively unambiguous and associated with general agreement between different authors regarding its contribution to ligand-NA binding energetics.

\section{The contribution from charge-transfer interactions}

The possibility of charge-transfer interactions in ligand-NA complexes has long been considered as one of the important sources of stabilization of the complexes and the CT is even considered by some authors (e.g. Ref. [111]) as a mean to create new drugs with increased affinity to DNA. To the best of our knowledge with respect to ligand-NA interactions in aqueous solutions (in conditions close to physiological) no solid experimental evidence to the existence of CT was reported. In Refs. [74,112] the probability of electron transfer from DNA to ellipticine, daunomycin, DAPI, ethidium bromide and its derivatives was estimated from quantum-mechanical calculations. It was shown that this probability is small (the amount of transferred charge $<0.2 e$ ), and the energy effect of this process, $\Delta G_{\mathrm{CT}}$, may be estimated only indirectly. Similar situation is the case for complexation between small molecules in aqueous solutions, which possess or do not possess opposite donor-acceptor properties. Under non-physiological conditions (low temperatures, organic solvents etc.) the spectroscopic evidence of CT was reported in complexes of small aromatic molecules (e.g. Refs. $[113,114])$ with very few works demonstrating the probability of CT under ambient temperatures and water solution (e.g. Refs. [115,116]). Quantum-mechanical and thermodynamic 
analyses performed for various complexes of aromatic molecules (e.g. Refs. [117-120]), including DNA intercalators, did not result in apparent evidence of CT contribution.

The indirect literature data given above suggest that under conditions of experiment close to physiological the contribution from CT interactions in ligand-NA complexes does not play significant role in energetics of binding and in majority of papers dealing with energy decomposition the problem is ignored.

\section{The contribution from specific entropic factors}

As already discussed in Section 4, specific entropic factors, such as the loss/formation of degrees of freedom on complexation is usually considered as a separate term and is sometimes related to more general entropic quantity, $\Delta G_{\text {config, originating from the }}$ change in configurational entropy, readily available from molecular dynamics simulations (e.g. Refs. [121-123]). However, the separation and comparative analysis of entropic terms for ligand-NA interactions appears to be a difficult task because different authors use different approaches to decompose and calculate these terms, and these results are often not simple to link together.

Energetic equivalent of the loss of translational and rotational degrees of freedom on ligand binding with DNA is taken by some authors equal to $\Delta G_{\mathrm{r}+\mathrm{t}}=63 \mathrm{~kJ} \cdot \mathrm{mol}^{-1}\left(15 \mathrm{kcal} \cdot \mathrm{mol}^{-1}\right)$ in Eq. (2), although the change in vibrational degrees of freedom is often not considered (e.g. Refs. [42-44,46-48]). This value was obtained for protein complexes by the methods of statistical thermodynamics [124,125]. In Ref. [49] this value was taken as $\Delta G_{\mathrm{r}+\mathrm{t}}=17 \mathrm{~kJ} \cdot \mathrm{mol}^{-1}\left(4 \mathrm{kcal} \cdot \mathrm{mol}^{-1}\right)$ based on the method of effective and angular volumes [126], which is different from the previous value, and the vibrational contribution was also not considered. In the rest of the works available which may be directly used in the context of the energy of entropic contribution, e.g. Refs. $[68,123,127,128]$, the contribution from vibrational degrees of freedom are included into the total change in configurational entropy which is computed from normal mode analysis (e.g. the Schlitter method [129]). In particular, in Ref. [127] for DNA-binding ligands the $\Delta G_{\text {config }}$ was estimated as $-T \Delta S_{\text {config }}=(29 \ldots 117) \mathrm{kJ} \cdot \mathrm{mol}^{-1}\left\{(7.8 \ldots 28) \mathrm{kcal} \cdot \mathrm{mol}^{-1}\right\}$ depending on the nucleotide sequence. The drawback of such calculations is the dependence of the value of $\Delta G_{\text {config }}$ on the simulation time $[68,123,127,128]$ (the higher the simulation time, the larger the number of microstates accounted for in calculations).

An important part of the overall entropic contribution, which may be decomposed as a separate term, or included into $\Delta G_{\text {config, }}$ or included into $\Delta G_{\text {conf }}$ term in Eqs. (2) and (8), is the change in NA/ligand conformation on binding. An approximation often used in this analysis is the consideration of the change in conformational entropy of the ligand only, ignoring DNA. For instance, the energy analysis of intercalating binding often does not take into account the $\Delta G_{\text {conf }}$ change of the ligand and fully associates it with DNA (e.g. Refs. $[26,123]$ ), whereas, in contrast, the MGB-binding with DNA considers the conformation of the ligand and sometimes ignores DNA (e.g. Refs. $[25,68])$. The typical values of $-T \Delta S_{\text {conf }}$ excluding DNA were obtained as (31 and 38$\left.) \mathrm{kJ} \cdot \mathrm{mol}^{-1}\right)\{(7.4$ and $\left.9) \mathrm{kcal}^{\circ} \mathrm{mol}^{-1}\right\}$ for distamycin and netropsin, respectively (e.g. Ref. [68]), whereas the net effect (including DNA and the ligand) appears to be higher, e.g. $-T \Delta S_{\text {conf }}=107 \mathrm{~kJ} \cdot \mathrm{mol}^{-1}\left\{25.6 \mathrm{kcal} \cdot \mathrm{mol}^{-1}\right\}$ calculated for Hoechst-DNA [128]).

At this point it is worth noting that there is much controversy in the literature regarding the extent of conformational change of the MGB ligand and DNA during their complexation. In the majority of works the conformational changes of both the DNA receptor and the MGB ligand are assumed to be negligible $[44,46-48,68,69]$.
The latter is confirmed by successful application of the single trajectory method taking implicit account of the water environment in the molecular dynamics simulations of the total energy of binding [127]. Within this approach the MD procedure is performed once for the ligand-DNA complex followed by extraction of the trajectories of the free molecules, which is equivalent to the assumption that the conformations of the DNA and the MGB ligand in the bound and unbound state are similar. However, in other approaches the calculated energy of conformational change may reach $50 \mathrm{~kJ} \cdot \mathrm{mol}^{-} 1\left(12 \mathrm{kcal} \cdot \mathrm{mol}^{-1}\right)$ [13], which is a significant contribution to the total Gibbs energy change. Insignificant changes in the twist, $\Omega$, and propeller, $\omega$, angles for DNA base pairs on binding with Hoechst33258 were inferred from NMR spectroscopy [130], although the energy effect of these changes remains unknown. In Ref. [25] it was shown that the value of the change in MGBligand conformational energy on complexation does not exceed $9 \mathrm{~kJ} \cdot \mathrm{mol}^{-1}\left(2 \mathrm{kcal} \cdot \mathrm{mol}^{-1}\right)$. This result is in agreement with similar estimations for other compounds, similar by structure and molecular weight (see, for example, $[70,131]$ ), suggesting that for the group of MGB ligands, the contribution from conformational restriction is insignificant. It should, however, be noted that large ligands with bulky side chains and numerous possibilities for internal rotations may, in principle, provide significant conformational contribution to the total Gibbs energy for the MGB ligands binding with DNA.

To the best of our knowledge in energy analyses of ligand binding with RNA the entropic contribution has not been considered explicitly (e.g. [132-134]). In Ref. [52] the net entropic contribution was qualitatively estimated as $\Delta G_{\text {entr }}=84 \mathrm{~kJ} \cdot \mathrm{mol}^{-1}$ $\left(20 \mathrm{kcal} \cdot \mathrm{mol}^{-1}\right)$. In Ref. [62] the sum of translational and rotational contribution was estimated as $(29 . .38) \mathrm{kJ} \cdot \mathrm{mol}^{-1} \quad\{(7 \ldots 9)$ $\left.\mathrm{kcal} \cdot \mathrm{mol}^{-1}\right\}$, the value for vibrational term was reported to be the same, i.e. (29...38) $\mathrm{kJ} \cdot \mathrm{mol}^{-1}$. The energetic cost of conformational change of RNA receptor on ligand-RNA binding is currently difficult to ascertain based on published material. Some authors do not take it into account (e.g. [52,62]), or implicitly account it as an 'adaptation' energy $\Delta G_{\text {conf }}=73 \mathrm{~kJ} \cdot \mathrm{mol}^{-1}\left(17.4 \mathrm{kcal} \cdot \mathrm{mol}^{-1}\right)$ [54]) or try to estimate it explicitly, e.g. $\Delta G_{\text {conf }}=(42 \ldots 159) \mathrm{kJ} \cdot \mathrm{mol}^{-1}\{(10 \ldots 19)$ $\left.\mathrm{kcal} \cdot \mathrm{mol}^{-1}\right\}[63,135]$.

One of the most general conclusions coming out from these studies was that the value of $\Delta G_{\text {entr }}$ or its terms commensurate with experimental energy of ligand-NA binding by absolute value, $\Delta G_{\text {entr }} \approx-42 \mathrm{~kJ} \cdot \mathrm{mol}^{-1}\left(-10 \mathrm{kcal} \cdot \mathrm{mol}^{-1}\right)$, being, however, always unfavourable in the net energy profile, indicating that this term must always be taken into account in energy analyses.

A systematic investigation of the entropic contribution to the Gibbs energy of ligand binding with DNA and RNA was accomplished in series of works $[25,26,64-66,136]$ according to the method based on Eq. (10) (Table 4). In all systems studied the quantities $\Delta G_{\mathrm{t}}$ and $\Delta G_{\mathrm{r}}$ were unfavourable for binding, which is due to the entropically unfavourable loss of three translational and rotational degrees of freedom upon complexation. The mean values of $\Delta G_{\mathrm{t}}$ and $\Delta G_{\mathrm{r}}$ averaged over the ligands studied within each of three groups, i.e. ligand-DNA intercalation, minor-groove binding and RNA-binding, fall within the same range of values, viz. $\left\langle\Delta G_{\mathrm{t}}\right\rangle=(39 \ldots 50) \mathrm{kJ} \cdot \mathrm{mol}^{-1}\left\{(9 \ldots 12) \mathrm{kcal} \cdot \mathrm{mol}^{-1}\right\}$ and $\left\langle\Delta G_{\mathrm{r}}\right\rangle=$ $(39 . .50) \mathrm{kJ} \cdot \mathrm{mol}^{-1}\left\{(9 \ldots 12) \mathrm{kcal} \cdot \mathrm{mol}^{-1}\right\}$. There is an agreement of these values between different research groups (e.g. [13,61]) which used similar approach. Thus, the differences in these energies for the different types of ligand are relatively small and so the mean energies $\left\langle\Delta G_{\mathrm{t}}\right\rangle$ and $\left\langle\Delta G_{\mathrm{r}}\right\rangle$ can effectively be used in analysis of the contributions for different small molecules complexing with NA. The mean sum $\left\langle\Delta G_{\mathrm{t}}+\Delta G_{\mathrm{r}}>\approx 84 \mathrm{~kJ} \cdot \mathrm{mol}^{-1}\left(20 \mathrm{kcal} \cdot \mathrm{mol}^{-1}\right)\right.$, is close to but slightly higher than the empirical value $\Delta G_{\mathrm{t}+\mathrm{r}}=63$ $\mathrm{kJ} \cdot \mathrm{mol}^{-1}\left(15 \mathrm{kcal} \cdot \mathrm{mol}^{-1}\right)$, used before $[19,42]$ for energy partitioning of ligand-DNA interactions. 
Table 4

Entropic energies for ligand binding with nucleic acids. The standard deviations are given in parentheses Entropic energy/4.18 kJ.mol ${ }^{-1}$.

\begin{tabular}{|c|c|c|c|c|c|c|}
\hline Type of complexation & $\begin{array}{l}\text { Translational } \\
\text { entropy }\end{array}$ & $\begin{array}{l}\text { Rotational } \\
\text { entropy }\end{array}$ & $\begin{array}{l}\text { Vibration of } \\
\text { chemical bonds }\end{array}$ & $\begin{array}{l}\text { Low-frequency vibration } \\
\text { of the ligand }\end{array}$ & $\begin{array}{l}\text { Net entropic } \\
\text { contribution }\end{array}$ & Refs. \\
\hline DNA intercalation & $\begin{array}{l}10.2 \\
(0.5)\end{array}$ & $\begin{array}{l}9.6 \\
(1.0)\end{array}$ & $\begin{array}{l}-4.3 \\
(0.3)\end{array}$ & $\begin{array}{l}-7.9 \\
(0.7)\end{array}$ & $\begin{array}{l}7.6 \\
(1.4)\end{array}$ & {$[26,42-44,46-48]$} \\
\hline DNA minor-groove binding & $\begin{array}{l}10.1 \\
(0.2)\end{array}$ & $\begin{array}{l}10.1 \\
(0.5)\end{array}$ & $\begin{array}{l}9.8 \\
(5.5)\end{array}$ & $\begin{array}{l}-7.9 \\
(0.2)\end{array}$ & $\begin{array}{l}22.1 \\
(6.0)\end{array}$ & {$[25,68]$} \\
\hline RNA aptamer complexation & $\begin{array}{l}10.1 \\
(0.4)\end{array}$ & $\begin{array}{l}9.6 \\
(0.7)\end{array}$ & $\begin{array}{l}22.1 \\
(10.4)\end{array}$ & $\begin{array}{l}-7.5 \\
(0.3)\end{array}$ & $\begin{array}{l}34.3 \\
(11.2)\end{array}$ & {$[54,66]$} \\
\hline
\end{tabular}

The energy change due to alteration in the mode of vibration of chemical bonds on complexation of MGB-ligands with DNA [25] and the ligands with RNA [66], $\Delta G_{\mathrm{v}}^{\mathrm{I}}$, is dominated by the enthalpic term, $\Delta H_{\mathrm{v}}^{\mathrm{I}}$, and is energetically unfavourable, indicating the absorbance of energy by newly-created vibrational modes induced by the complexation process. Interestingly, contrary to that the binding of aromatic intercalators with DNA was characterized by a favourable contribution of the $\Delta G_{\mathrm{v}}^{\mathrm{l}}$ term and predominantly entropic character of the binding $[26,64,136]$. For flexible molecules and/or binding sites, the $\Delta G_{\mathrm{v}}^{\mathrm{l}}$ term may take both negative or positive sign as also demonstrated for protein-protein and proteinDNA interactions (see Ref. [83] and references therein). Comparative analysis of the $\Delta G_{v}^{1}$ term between different research groups is difficult since the majority reports consider the vibrational contribution for NA-binding ligands to be either implicitly included into the total configurational entropy change of the system (e.g. Refs. $[68,127,128])$, ignored all together (e.g. Refs. [44,46-48]) or considered negligible (e.g. Refs. [61]).

The energy change, $\Delta G_{v}$, resulting from the appearance of new low frequency vibrational modes of the ligand as a whole within the binding site is always favourable for ligand-NA systems studied and is dominated by the entropic term, which is the result of creating new vibrational degree of freedom due to the appearance of mechanical oscillation of a ligand on binding $[25,26,64,136]$. The independent justification for the existence of the $\Delta G_{\mathrm{v}}^{\mathrm{II}}$ term in the structure of the total Gibbs energy of complexation reactions comes from ultrafast fluorescence monitoring of the dynamics of ligand-DNA complexes [137], which indirectly confirms the existence of mechanical oscillations of the ligand within the binding site.

In total, for the majority of the NA-binding ligands studied, the $\Delta G_{\mathrm{v}}^{\mathrm{I}}$ and $\Delta G_{\mathrm{v}}^{\mathrm{II}}$ terms nearly compensate each other and the net entropic contribution to the Gibbs energy of their binding with DNA is mainly determined by the sum, $\Delta G_{t}+\Delta G_{\mathrm{r}}$. As a result, the net entropic contribution, $\Delta G_{\text {entr, }}$, for ligand binding with DNA/ RNA is unfavourable. Specifically for the group of aromatic intercalators the $\Delta G_{\text {entr }}$ term was found to differ little from the mean value and the total sum $\left\langle\Delta G_{\text {entr }}\right\rangle=33.6 \mathrm{~kJ} \cdot \mathrm{mol}^{-1}\left(7.8 \mathrm{kcal} \cdot \mathrm{mol}^{-1}\right)$ was suggested for the use in the energy analysis of ligand-DNA complexation for different aromatic ligand with non-heavily branched side chains $[26,64]$.

One final point regarding the entropic factors is worth mentioning. Recently the hidden entropic factors contributing to the $\Delta G_{\text {total }}$ were revealed [138-140], originating from dependence of microscopic binding constant, $K$, on concentration of the ligand. This effect is due to the intrinsic dependence of $\Delta G_{t}$ and $\Delta G_{\mathrm{r}}$ on the mass of the receptor and the dependence of the rigidity of DNA molecule on the number of bound ligands. It was, however, shown, that both effects are negligible when dealing with polymeric DNA and the ligands which have negligible dimensions with respect to DNA (i.e. the 'small molecule' approach).

\section{The general energy analysis of ligand-NA binding interactions}

\subsection{Requirements to accomplishing the full energy analysis}

The computation and analysis of various terms of the total Gibbs energy, reviewed above, enables to approach the main goal of energy analysis, formulated in the introductory section, viz. 'What physical factors and to what extent stabilize/destabilize ligand-NA complexes?' and 'What physical factor most highly correlates with the parameter of interest? (e.g. the complexation constant or biological activity)'. It was proposed $[14,25,26]$ that these principal tasks of energy analysis can be solved, if a protocol for computation of the contributions to the energies of the principal energy terms (or parsing/decomposition of the Gibbs energy) satisfies the following conditions:

(i) summation of the independently-calculated energy terms (according to equations given in Section 4) reproduces the experimentally-measured total energy of interaction within reasonable error limits. In that case the calculated energies for various physical factors are meaningful and so these energies may be used in comparative analysis;

(ii) the calculations should be applied to a set of molecular systems that differ in structure and charge state. If the protocol only demonstrates satisfactory coincidence with experiment for a single system (as is often the case), the transferability to other systems will always be questionable, hence, there is no guarantee that the calculated energies are generally meaningful;

(iii) the calculations should be made using a similar protocol and set of parameters/restraints for each system studied. Otherwise, it appears that there may be an artificial adjustment to the results, making the calculated energies less reliable.

This set of points may be considered as the requirements for meaningful energy analysis.

So far the agreement between the $\Delta G_{\text {total }}$ and $\Delta G_{\exp }$ for the ligand-NA binding reactions has been reached in very few cases (e.g. Refs. $[25,26,59,66,141])$ of which, to the best of our knowledge, the full compliance with the given list of requirements has been reported only in Refs. [25,26,66]. Below we shall mainly use these results in reviewing the general outcome of the energy analysis.

\subsection{Intrinsic error in computation of the total binding energy and meaningfulness of the energy analysis}

Prior to conducting the full energy analysis, it is important to verify that the theoretically computed according to Eqs. (2), (4), (6)-(9) total energies, $\Delta G_{\text {total }}$, match the experimentally measured energy, $\Delta G_{\text {exp }}$. 
$\Delta G_{\text {total }}$ is formed as a sum of various separately computed terms, each of which contributes its own error to the net energy being the largest for the electrostatic, van der Waals and hydrophobic terms as the most important ones. Within the methodology of the energy decomposition based on the use of the methods of molecular modeling, the sources of the principal errors are the accuracy of representation of the van der Waals parameters in applied force field specifically for the group of ligands studied, implicit or explicit account of the polarization effects inside the binding site and hidden in the electrostatic term, the accuracy of representation of hydrophobic interactions in eqns.3,5,11 etc. Although the computation of the exact error of the binding energy is difficult to make, it is generally considered that for each of the three principal terms it should be an order of few kJ. $\mathrm{mol}^{-1}$ (see Ref. [67] and references therein for more discussion). Hence, the total estimated error in the computation of $\Delta G_{\text {total }}$ for ligand-NA interactions was reported to be within the range $<42 \mathrm{~kJ} \cdot \mathrm{mol}^{-1}\left(10 \mathrm{kcal} \cdot \mathrm{mol}^{-1}\right)[25,26,37]$. It follows that the mean difference, $\left\langle\left|\Delta G_{\text {total }}-\Delta G_{\text {exp }}\right|\right\rangle$, between the experimental and calculated energies is lost within the error of $\Delta G_{\text {total }}$ determination. It means that the decomposition of the total Gibbs energy for structurally different NA-binding ligands must be considered successful if the difference between $\Delta G_{\text {total }}$ and $\Delta G_{\text {exp }}$ for each particular ligand falls within the range
$\left|\Delta G_{\text {total }}-\Delta G_{\text {exp }}\right| \in 0 \ldots \Delta G_{\text {exp }}$

Such view on the error of $\Delta G_{\text {total }}$ computation has to date been implicitly or explicitly stated by different researches (e.g. $[25,26,60])$. Its most important outcome is the acknowledgement that $\Delta G_{\text {total }}$ is an unreliable quantity and should not be used in energy analysis of ligand-NA interactions. However, the separate energy terms making up $\Delta G_{\text {total }}$ and calculated with higher accuracy, bear physical meaning and can be used in energy analyses. The agreement with Eq. (16) was reached for different DNA intercalators [26,49,64], MGB-ligands [25,59,141] and RNA-binders [66]. Once the agreement between the experimental and calculated total energies has been established, it is further possible to analyse the computed energies with an aim of searching general patterns in the distribution of energy over various terms, i.e. to start answering the two principal questions of energy analysis formulated in Section 2.

\subsection{What physical factors and to what extent stabilize/destabilize complexes of small molecules with NA?}

An example of Gibbs energy decomposition for the three groups of NA-binding ligands discussed in the present review (viz. intercalators, MGB- and RNA-binders) is given in Fig. 2 in the form of two
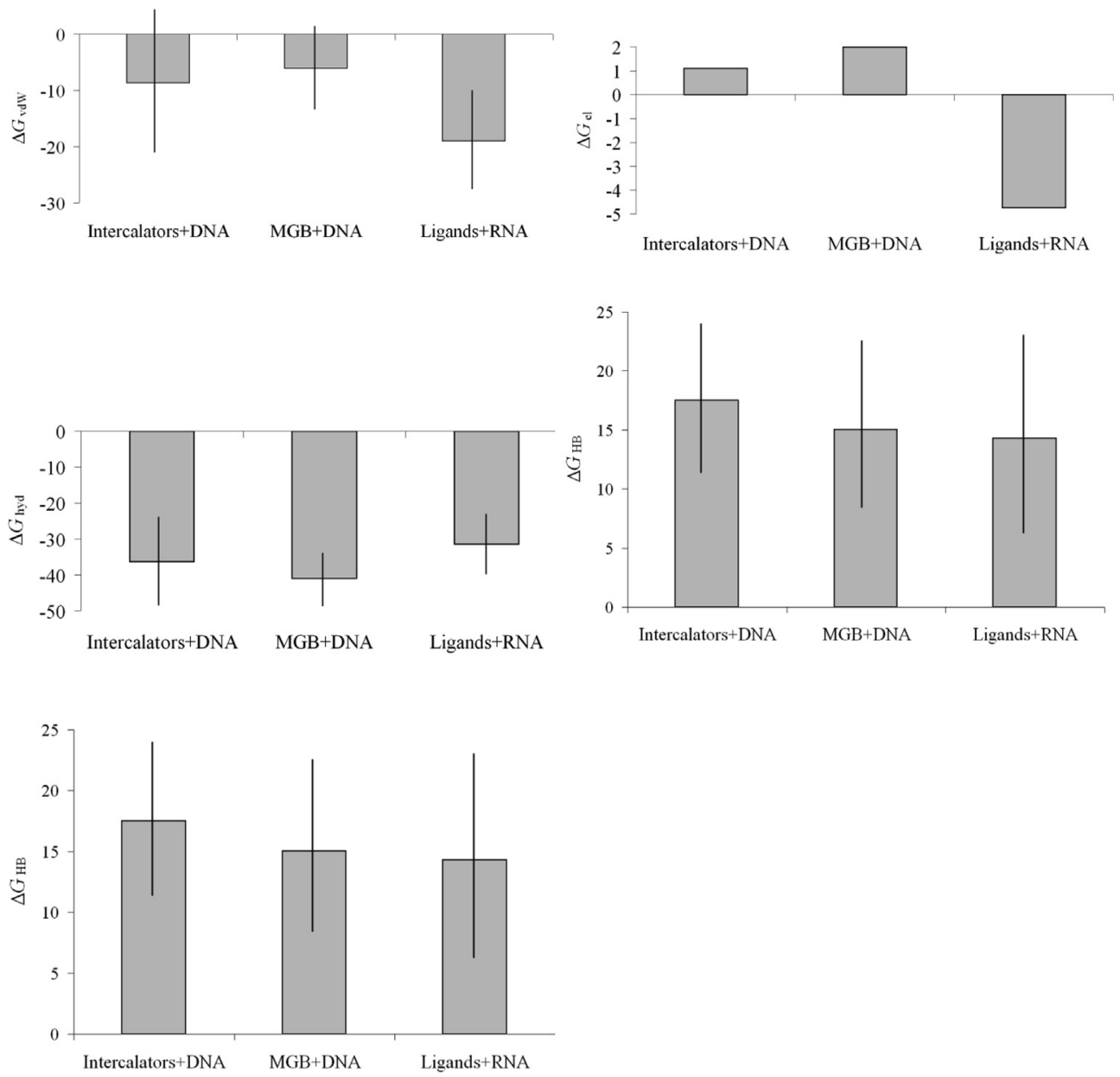

(a)

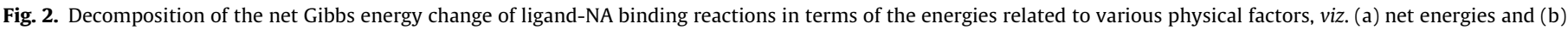
component energies. 

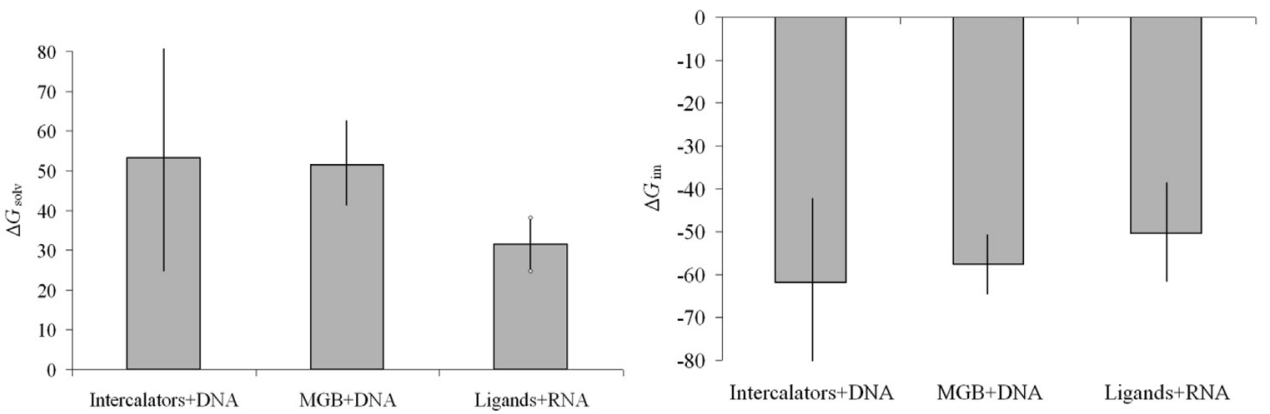

Van der Waals
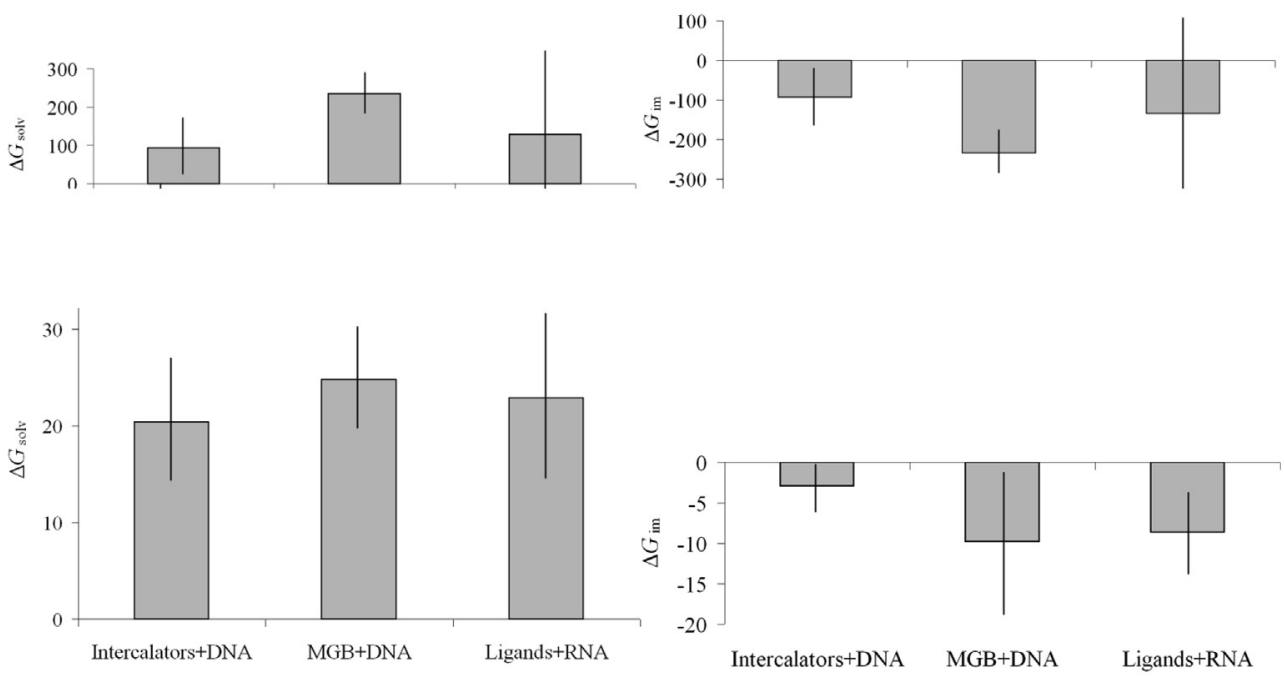

Hydrogen bonds
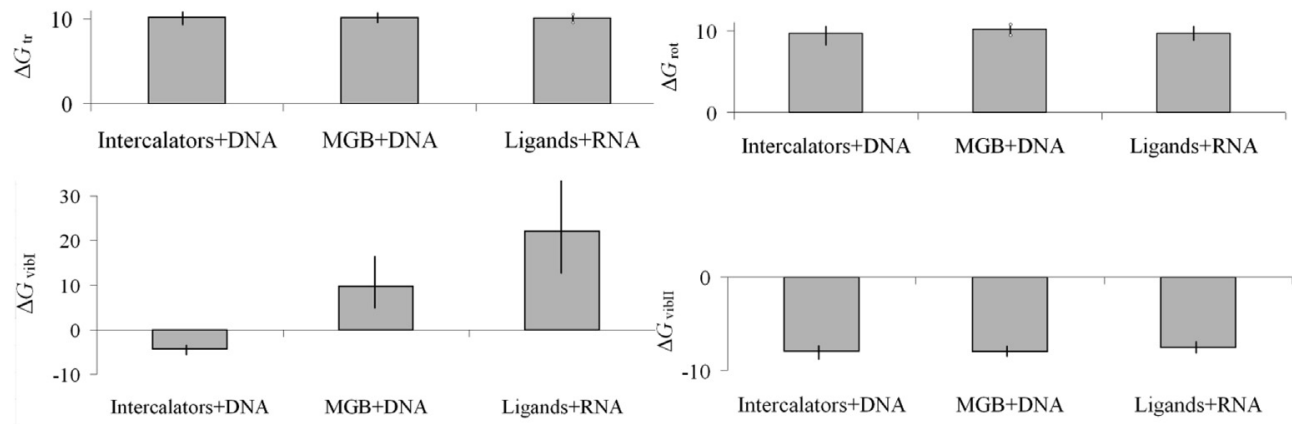

Entropic

(b)

Fig. 2 (continued)

different strategies of decomposition, viz. the decomposition in terms of the net energies (Eq. (2) or Eq. (8)) **and in terms of component energies (Eq. (9)), related to various physical factors, computed in $[25,26,64,66]$.

It follows from Fig. 2 that within the set of the net energies the most important stabilizing factors are hydrophobic interactions. Van der Waals, polyelectrolyte and electrostatic factors give relatively small stabilization and destabilization of the complexes, respectively, as a result of summation of the 'im' and 'solv' terms larger by value and opposite by sign. As a consequence, the net electrostatic energy may take either zero, small positive and negative numbers, reflecting the error in computations rather than some pattern. The entropic term, $\Delta G_{\text {entr }}$, related to the net changes in the number of degrees of freedom, and the hydrogen bond term, related to the net loss of $\mathrm{H}$-bond contacts of the interacting molecules, $\Delta G_{\mathrm{HB}}$, are unfavourable. This pattern is similar for both the DNA intercalative binding and MGB- or RNA-binding, and was also previously reported for the interaction of aromatic ligands with each other [67]. Such analysis, although being quite natural and being used on occasions in the literature (e.g. [44,46-48]), provides 
little understanding of the set of factors that stabilize ligand-NA complexes. For example, it appears that the hydrophobic contribution is always dominant and the net electrostatic and van der Waals interactions may be relatively unimportant or even take nearly zero values (e.g. [42]). Such energy analysis is considered meaningless and the net energies associated with the contribution of each particular physical factor typically do not demonstrate correlation with physical properties of the ligands, viz. the ligand dimensions, type of side chains and electric charge [25,26,88]. It was suggested $[14,25-27,88]$ that a real understanding of the major stabilizing factors may only come from analysis of the components of the net energies related to each particular physical factors and type of interaction (i.e. with solvent or intermolecular). Let us consider the energy decomposition in terms of components of the net energies for each particular group of NA-binding ligands.

\subsubsection{DNA intercalative binding}

It is seen (see Fig. 2) that the DNA unwinding stage is always unfavorable for ligand binding and is the main contributor to the activation energy for the reaction of ligand-DNA complexation.

The major stabilization comes from intermolecular interactions on the stage of insertion: electrostatics and, to a lesser extent VDW, followed by $\Delta G_{\mathrm{hyd}}>\Delta G_{\mathrm{HB}}^{\mathrm{im}}>\Delta G_{\mathrm{pe}}$ placed in descending order by absolute value. The factors which destabilize ligand-DNA complexes can be placed in an order: $\Delta G_{\mathrm{el}}^{\text {solv }}>\Delta G_{\mathrm{vdW}}^{\text {solv }}>\Delta G_{\mathrm{HB}}^{\text {solv }}>\Delta G_{\text {entr }}$.

\subsubsection{RNA binding}

It is seen (see Fig. 2) that the stabilizing energy terms can be placed in the sequence by extent of their contribution: $\Delta G_{\mathrm{vdW}}^{\mathrm{im}}>\Delta G_{\mathrm{hyd}}>\Delta G_{\mathrm{v}}^{\mathrm{II}}$. The sequence for the destabilizing energies is: $\Delta G_{\mathrm{vdW}}^{\text {solv }} \geq \Delta G_{\mathrm{v}}^{\mathrm{l}} \geq \Delta G_{\mathrm{t}} \approx \Delta G_{\mathrm{r}}$. The van der Waals energies were found to depend strongly on the type and dimensions of side chains of the ligand and the efficacy of $\pi$-stacking with RNA bases. It is difficult to include the electrostatic energy into this set as it demonstrates big dispersion depending on the type of ligand, which was not so pronounced for the case of MGB- and intercalative bindings. As already discussed above, the intermolecular $\left(\Delta G_{\mathrm{el}}^{\mathrm{im}}\right)$ and to-water $\left(\Delta G_{\mathrm{el}}^{\mathrm{solv}}\right)$ electrostatic energies by the value and sign strongly depend on the charge of the ligand and, to lesser extent, on ligand dimensions, giving either predominant or not predominant contribution to $\Delta G_{\text {total }}$ as compared with other energy terms. The RNA-binding is probably the best example to demonstrate the inapplicability of energy analysis based on net energies (see above) which removes the correlation of the energy with physical properties of the ligand and/or binding site on summation of the 'solv' and 'im' terms.

\subsubsection{DNA minor-groove binding}

It is seen (see Fig. 2) that there are at least three major stabilizing factors which govern the binding process of MGB ligands with DNA, placed in descending order according to the absolute value of energy change: intermolecular electrostatic interactions $\left(\Delta G_{\mathrm{el}}^{\mathrm{im}}\right)$, intermolecular van der Waals interactions $\left(\Delta G_{\mathrm{vdW}}^{\mathrm{im}}\right)$ and hydrophobic interactions $\left(\Delta G_{\text {hyd }}\right)$. It is worth noting that the electrostatic stabilization of MGB-DNA complexes was reported by various authors (e.g. Refs. [13,25,142]), although a direct comparison of the electrostatic energy with other factors can only be made in full energy analysis. The stabilization of the complexes is also provided by the formation of intermolecular H-bonds $\left(N^{\mathrm{im}}\right)$, formation of residual mechanical vibrations in the binding site $\left(\Delta G_{v}^{\mathrm{II}}\right)$ and the polyelectrolyte factor $\left(\Delta G_{\mathrm{pe}}\right)$ - the latter two giving minor contribution as compared to other factors.

An estimation of the contribution of $\Delta G_{\mathrm{HB}}^{\mathrm{im}}$ term was given in Ref. [25] as $\Delta G_{\mathrm{HB}}^{\mathrm{im}}=-3 \cdot N^{\mathrm{im}}=-(3 \ldots 33) \mathrm{kcal} / \mathrm{mol}$ depending on the num- ber of intermolecular H-bonds, $N^{\mathrm{im}}$. It was concluded that the energy of intermolecular $\mathrm{H}$-bonding gives relatively small contribution to the energetics of MGB binding with DNA.

The complete set of the factors which stabilize MGB-DNA complexes, can be written in the following order by the absolute value of the stabilizing energy [25]: $\Delta G_{\mathrm{el}}^{\mathrm{im}}>\Delta G_{\mathrm{vdW}}^{\mathrm{im}}>\Delta G_{\mathrm{hyd}}>\Delta G_{\mathrm{v}}^{\mathrm{Il}}>\Delta G_{\mathrm{pe}}$.

The major factors which destabilize complexes of the MGB ligands with DNA are the electrostatic $\left(\Delta G_{\mathrm{el}}^{\text {solv }}\right)$ and van der Waals $\left(\Delta G_{\mathrm{vdW}}^{\text {solv }}\right)$ desolvation, loss of H-bonds to-water $\left(\Delta N^{\text {solv }}\right)$, change in the number of translational $\left(\Delta G_{\mathrm{t}}\right)$, rotational $\left(\Delta G_{\mathrm{r}}\right)$, vibrational $\left(\Delta G_{\mathrm{v}}^{\mathrm{l}}\right)$ degrees of freedom, and restriction of internal rotations in MGB molecules $\left(\Delta G_{\text {conf }}\right)$. The set of destabilizing factors were placed in an order: $\Delta G_{\mathrm{el}}^{\text {solv }}>\Delta G_{\mathrm{vdW}}^{\text {solv }}>\Delta G_{\mathrm{HB}} \geq \Delta G_{\mathrm{t}} \approx \Delta G_{\mathrm{r}} \approx \Delta G_{\mathrm{v}}^{\mathrm{l}}>\Delta G_{\text {conf. }}$

\subsubsection{The general patterns in energy terms}

The review of energy analysis over particular energy terms analyzed in separate (Sections 5 to 11) and all together (Section 12) allows some general conclusions to be drawn.

It can be seen that ligand binding to DNA is governed by the effect of compensation of energy contributions at the levels of physical factors, different stages of ligand binding and inter(intr a)molecular/to-solvent interactions in vacuum. The consequences of this compensation are the following [88]:

- summation of large by value (dozens-hundreds $\mathrm{kcal} / \mathrm{mol}$ ) energy terms, associated with the contribution of various physical factors into $\Delta G_{\text {total }}$ (Eqs. (2), (8), (9)), results in relatively small experimental Gibbs energy of binding (i.e. $\Delta G_{\text {exp }}$ $\sim 42 \mathrm{~kJ} \cdot \mathrm{mol}^{-1}\left(10 \mathrm{kcal} \cdot \mathrm{mol}^{-1}\right)$;

- de-solvation of the ligand and NA on complexation, characterized by large positive energies of interaction with solvent, is compensated by negative energies of intermolecular interactions in the complex;

- depending on the electric charge of the ligand and NA the value of the electrostatic energy of interaction can take large values (hundreds of $\mathrm{kJ} \cdot \mathrm{mol}^{-1}$ for the type of interacting entities ' + ' and '-', and units of $\mathrm{kJ} \cdot \mathrm{mol}^{-1}$ for the interaction of neutral entities). However, the net electrostatic $\left(\Delta G_{\mathrm{el}}\right)$ and experimental $\left(\Delta G_{\text {exp }}\right)$ energies are always small and in majority of cases both do not exert correlation with the charge of interacting molecules.

The compensatory origin of experimental binding Gibbs energy, presumably, reflects the general pattern of binding reactions in aqueous media $[19,49,88,143]$ and has so far been reported for ligand-protein interactions as well $[12,18,60,83]$.

\subsection{What physical factor most highly correlates with the parameter} of interest?

The set of stabilizing and destabilizing energies aligned in descending order, as the main outcome of the energy analysis, provides a fundamental knowledge on energetics of binding reactions in solution but, in fact, gives little idea on the way how one can manipulate the $\Delta G_{\text {exp }}$ and, eventually, the medico-biological effect of the NA-binding drugs. It is considered that the search of the factor which is most strongly correlated with the equilibrium binding constant $K=\exp \left(-\frac{\Delta G_{\exp }}{R T}\right)$ may give this idea [14,25,26]. If it is known what factor modulates the ligand affinity to DNA (van der Waals, hydrophobic, electrostatics or else), it may give an idea what type of atomic group must be chemically added/substituted in the ligand structure in order to amplify the contribution of this particular physical factor to the net energy of binding, resulting in increase of $\Delta G_{\text {exp. }}$. Let us further consider the solution 
of this task with respect to NA-binding ligands discussed in the present review.

\subsubsection{DNA intercalative binding}

Search of a correlation ( $r$ ) of the energy terms in Eq. (9) with the experimental energy, $\Delta G_{\text {exp }}$, has shown $[26,64,88]$ that the highest impact on it is provided by the VDW energy of ligand insertion, $\Delta G_{\mathrm{vdW}}^{\mathrm{ins}}(r=0.66)$ and VDW energy of DNA unwinding, $\Delta G_{\mathrm{vdW}}^{\mathrm{iw}}(r=-0.67)$. The rest terms give lower correlation not exceeding $|r|=0.5$. The correlation between $\Delta G_{\exp }$ and $\Delta G_{\text {hyd }}$ equals to $r=0.42$. Noteworthy, the EL energy, formally featuring the largest contribution from the energy components (see Table 2), appears to be relatively unimportant in the modulating the binding affinity in the intercalation reactions. This result highlights the key role of the intermolecular VDW forces in managing the affinity of aromatic drugs to DNA and points out the way to modify the ligand structure with an aim to increase the binding strength with DNA.

\subsubsection{RNA binding}

The correlation coefficients of the terms in Eq. (9) with $\Delta G_{\exp }$ do not exceed 0.5 and do not allow selecting the terms which exert the maximal impact on the binding affinity [66,88]. It was suggested [66] that the principal physical factors, such as van der Waals, electrostatic, hydrophobic, hydrogen bonds, give approximately equal contribution to the variability of $\Delta G_{\exp }$ with the type of RNA-binding ligand.

\subsubsection{DNA minor-groove binding}

The correlation of the experimental energy with the solvation and intermolecular components for all the terms of the net Gibbs energy gives the highest value for the EL energy which suggests that the major effect on variation of $\Delta G_{\exp }$ with the type of ligand is provided by the electrostatic component [25]. The loss of $\mathrm{H}$ bonds to-water (the $\Delta N^{\text {solv }}$ term), was also reported to correlate with $\Delta G_{\text {exp }}$, is more closely related to electrostatics as well. This result identifies electrostatics as a major determinant of the difference between the studied MGB ligands in terms of their affinity to DNA. It was suggested $[25,88]$ that the electrostatic factor is probably what should be targeted in first instance when optimizing MGB affinity to DNA in rational drug design.

At this point it is worth recalling that the net $\Delta G_{\mathrm{el}}$ and $\Delta G_{\mathrm{vdW}}$ energies in Eq. (8) may be relatively small and the values commensurate with intrinsic error of the computation (see Tables 1,2), formally suggesting the relative unimportance of the electrostatic and van der Waals forces (see discussion in Sections 5, 6, 12). It is now seen that the conclusions concerning the nature of the factors stabilizing drug-DNA complexes may lead to completely different and erroneous result if the net energies are used in analysis. It is again worth repeating that only the analysis of component energies from Eq. (9) may give meaningful results.

\subsubsection{Correlation of the energy terms with biological activity}

The approach to answer the question 'What physical factor most highly correlates with the parameter of interest?' may be of value if the binding affinity is the target property to be manipulated, or if no sufficient data on biological activity of the studied group of ligands is available, and the amplification of the binding affinity to bioreceptor remains the only possible strategy. The case if relevant biological data are available, search of correlations between the biological activity and specific energy terms may have real practical outcome. Such possibility was considered in [14,88] taking as an example the group of MGB binders.

The $\mathrm{ID}_{50}$ factor (which is a micromolar concentration of the drug, needed for 50\% suppression of L1210 leukemia cell growth) measured for typical DNA minor-groove binding drugs was correlated with energy terms in Eq. (9). It was found that the highest correlation of the $\mathrm{ID}_{50}$ factor is the case with the change in the net number of hydrogen bonds on binding: $N^{\mathrm{im}}+\Delta N^{\text {solv }}$, hence, it is suggested that this factor might be modified in first instance in rational MGB-drug design. Interestingly, the $\mathrm{ID}_{50}$ factor did not show apparent correlation with the MGB-DNA binding constant (as an integral measure of the net energetics of binding), indicating that the use of $K$ as a parameter of interest (see above) not always results in proportional change in biological effect. Although this result was considered as preliminary, it clearly demonstrates the potential importance of the energy analysis in designing new drugs.

\section{The problems of energy analysis and future perspective}

Based on the above-performed review of the energetics of ligand-NA binding interactions, it is possible to highlight some difficulties and contradictory views, available in literature and providing the major challenge for future studies in this area. Although the selection of the method to compute the component energies and the level of correspondence of the obtained results to experiment will long be a matter of debate, here we shall deal with the principal points of energy analysis resulting in most strong qualitative discrepancies in views of different research groups, omitting the quantitative details of computing each particular terms of the net Gibbs energy, partly discussed in the sections above. At least three principal problems of energy analysis implicitly or explicitly existing in many publications in the field of ligandNA energetics are worthy of mentioning.

\subsection{The contribution from charge-transfer interaction}

As discussed in Section 10 the role of this type of interaction in ligand-NA binding energetic remains, in fact, unknown. As a consequence, this contribution may be considered as important or not important depending on the experimental method used and personal view of the researcher. However, the successful attempts to solve the energy decomposition problem for various NA-binding drugs ignoring the $\Delta G_{\mathrm{CT}}$ term, reviewed above, indirectly prove that this factor is likely of small importance in binding energetics in water solutions.

\subsection{Solution of the energy decomposition task for enthalpy, entropy and heat capacity changes}

The published material reviewed above proves the possibility to accomplish full energy analysis on the level of Gibbs energy change resulting in agreement with experiment for different by structure and charge state NA-binding ligands. The shortcoming of this approach is that the decomposition is made on the level of just one experimental value, $\Delta G_{\text {exp }}$, whereas the currently available biophysical methods allow measuring with high accuracy two other thermodynamic quantities, viz. the enthalpy, $\Delta H_{\text {exp }}$, and heat capacity, $\Delta C_{P}^{\exp }$, changes. To the best of our knowledge, the decomposition on the level of enthalpy and heat capacity for NA-binding reactions has to date not been accomplished except that of few papers $[42,144,145]$. Both quantities contain different by weight contribution from particular physical factors, enabling to explore them more comprehensively than that provided by $\Delta G_{\text {exp }}$, changing relatively insignificantly with the type of ligand studied, and formed by compensation of energetic contribution from various sources. Moreover, some published preliminary data point out that such decomposition may be difficult when attempting to meet the 
three requirements listed in Section 12.1 [145]. The key reasons of this negative outcome are thought to be $[22,27,50,145,146]$ :

(i) the difficulty to explicitly decompose enthalpic and entropic contributions into the energy terms of various physical factors,

(ii) much higher relative error in estimation of enthalpy as compared with $\Delta G$, originating from the compensatory nature of formation of Gibbs energy, and

(iii) insufficient level of elaboration of the theory and methods of computation of $\Delta C_{P}^{\exp }$.

Taking into account the difficulties mentioned above it is considered that the full utilization of the potential of energy analysis of studying the NA-binding reactions will only be achieved when the energy decomposition task is solved simultaneously on the levels of $\Delta G_{\text {exp }}, \Delta H_{\text {exp }}$ and $\Delta C_{P}^{\text {exp }}$. To best of our knowledge the similar task has not been solved for ligand-protein interactions as well, creating the strategy of future development of energy analysis of biomolecular interactions in solution.

\subsection{Contradictory view on involvement of water in ligand-NA interactions}

A strong controversy exists in the literature regarding the net effect of changes in hydration of NA-binding ligands on complexation with DNA. A group of methods based on structural studies $[13,25,26,95,147]$ and volumetry/densimetry [148-151] suggest that there is a net dehydration of the ligand and DNA molecules on complex formation (i.e. $\Delta N^{\text {solv }}<0$ ), whereas results from osmometry [152-155] lead to the opposite conclusion, viz. the binding of ligands results in an uptake of additional water molecules (i.e. $\Delta N^{\text {solv }}>0$ ). The recently published data based on ultrahigh frequency dielectrometry [156], unfortunately, did not provide an unambiguous answer to this question. This controversy is not specific to a particular group of ligands but holds for both the MGB-ligands and DNA intercalators. It was suggested [155,157] that volumetry and osmometry measure different types of bound water, viz. the strongly-bound water in volumetry and both, the strongly- and weakly-bound, in osmometry. It is, however, difficult to ascertain whether this view may explain the principal difference in the sign of the change in hydration, $\Delta N^{\text {solv }}$, in these two methods. Another view is the assumption on the cooperative character of the bound water [158], which may result in cooperative uptake of water molecules on ligand binding with DNA. However, no experimental evidence for this was provided. Finally, it was suggested [108] that the method of osmometry itself, when applied to ligand-DNA binding systems, may contain hidden errors associated with evaluation of the change in hydration of the solute molecules. Within the context of the present review it is worth noting that the separate terms constituting the total Gibbs energy, all reflect net dehydration of the ligand and the DNA as a result of the complexation process, viz. the desolvation of the solute molecules monitored by unfavourable change in van der Waals $\left(\Delta G_{\mathrm{vdW}}^{\text {solv }}\right.$, see Section 5$)$ and electrostatic ( $\Delta G_{\mathrm{el}}^{\text {solv }}$, see Section 6 ) energies of solvation, loss of hydrogen bonds to-water ( $\Delta N^{\text {solv }}$, see Section 8$)$ and the negative sign of the hydrophobic energy ( $\Delta G_{\text {hyd }}$, see Section 7$)$ indicating entropically favourable loss of solvent ordering. The issue on the involvement of water in ligand binding with nucleic acids is to be resolved in future studies.

\section{Concluding remarks}

The present review deals with energy analysis of ligand binding with nucleic acids in terms of the energy contribution from various physical factors making up the net Gibbs energy change of the complexation reaction measured in experiment. It is shown that the general patterns in distribution of energy over various energy terms may be correlated by either the structure of the ligand (type of side chains, type and number of aromatic rings etc.), or the binding affinity or biological activity. Thus, further manipulation of the leading energy factors by means of chemical modification of the drug provides an extension to scientific background of the strategy of rational drug design, as a supplementary to currently existing approaches. Its main outcome is the possibility to create new drugs with improved pharmacological properties, which currently remains one of most important challenges in biomedical sciences.

\section{References}

[1] K. Nakamoto, M. Tsuboi, G.D. Strahan, Drug-DNA interactions: structures and spectra, 51, John Wiley \& Sons, 2008.

[2] M.R. Reddy, M.D. Erion (Eds.), Gibbs Energy Calculations in Rational Drug Design, Springer US, 2001.

[3] A.N. Lane, T.C. Jenkins, Thermodynamics of nucleic acids and their interactions with ligands, Quart. Rev. Biophys. 33 (2000) 255-306.

[4] M. Demeunynck, C. Bailly, W.D. Wilson (Eds.), Small Molecule DNA and RNA Binders: From Synthesis to Nucleic Acid Complexes, Wiley-WCH, 2003.

[5] H. Ihmels, D. Otto, Intercalation of Organic Dye Molecules into DoubleStranded DNA - General Principles and Recent Developments, in Supermolecular Dye Chemistry, ed, Springer, Berlin Heidelberg, Frank Würthner, 2005, pp. 161-204.

[6] J.B. Chaires, Dissecting the free energy of drug binding to DNA, Anticancer Drug Des. 11 (1996) 569-580.

[7] P.L. Kastritis, A.M.J.J. Bonvin, On the binding affinity of macromolecular interactions: daring to ask why proteins interact, J. Royal Soc. Interface 10 (2013) 20120835.

[8] X.-L. Yang, A.H.-J. Wang, Structural studies of atom-specific anticancer drugs acting on DNA, Pharmacol. Therap. 83 (1999) 181-215.

[9] M.F. Brana, M. Cacho, A. Gradillas, B. de Pascual-Teresa, A. Ramos, Intercalators as anticancer drugs, Curr. Pharm. Des. 7 (2001) 1745-1780.

[10] J.O. Armitage, The role of mitoxantrone in non-Hodgkin's lymphoma, Oncology 16 (2002) 490-512.

[11] J.B. Chaires, F. Leng, T. Przewloka, I. Fokt, Y.-H. Ling, R. Perez-Soler, W. Priebe, Structure-based design of a new bisintercalating anthracycline antibiotic, J. Med. Chem. 40 (1997) 261-266.

[12] B. Jayaram, K. McConnell, S.B. Dixit, A. Das, D.L. Beveridge, Free-energy component analysis of 40 protein-DNA complexes: a consensus view on the thermodynamics of binding at the molecular level, J. Comput. Chem. 23 (2002) 1-14.

[13] S.A. Shaikh, S.R. Ahmed, B. Jayaram, A molecular thermodynamic view of DNA-drug interactions: a case study of 25 minor-groove binders, Arch. Biochem. Biophys. 429 (2004) 81-99.

[14] M.P. Evstigneev, A.V. Shestopalova, Structure, thermodynamics and energetics of drug-DNA interactions: computer modeling and experiment, Appl. Comp. Tech. Pharm. Med. (2014) 21-57, Springer, Netherlands.

[15] I. Haq, Thermodynamics of drug-DNA interactions, Arch. Biochem. Biophys. 403 (2002) 1-15.

[16] R. Perozzo, G. Folkers, L. Scapozza, Thermodynamics of protein-ligand interactions: history, presence, and future aspects, J. Recept. Signal Transduct. 24 (2004) 1-52.

[17] L. Jen-Jacobson, L.E. Engler, L.A. Jacobson, Structural and thermodynamic strategies for site-specific DNA binding proteins, Structure 8 (2000) 10151023.

[18] K.P. Murphy, Predicting binding energetics from structure: looking beyond $\Delta G^{0}$, Med. Res. Rev. 19 (1999) 333-339.

[19] J.B. Chaires, Energetics of drug-DNA interactions, Biopolymers 44 (1997) $201-215$.

[20] I. Haq, T.C. Jenkins, B.Z. Chowdhry, J. Ren, J.B. Chaires, Parsing free energies of drug-DNA interactions, Methods Enzymol. 323 (2000) 373-405.

[21] K.J. Breslauer, D.P. Remeta, W.-Y. Chou, R. Ferrante, J. Curry, D. Zaunczkowski, J.G. Snyder, L.A. Marky, Enthalpy-entropy compensations in drug-DNA binding studies, Proc. Natl Acad. Sci. U.S.A. 84 (1987) 8922-8926.

[22] K.A. Dill, Additivity principles in biochemistry, J. Biol. Chem. 272 (1997) $701-$ 704.

[23] P. Gilli, V. Ferretti, G. Gilli, P.A. Borea, Enthalpy-entropy compensation in drug-receptor binding, J. Phys. Chem. 98 (1994) 1515-1518.

[24] U. Ryde, A fundamental view of enthalpy-entropy compensation, Med. Chem. Comm. 5 (2014) 1324-1336.

[25] V.V. Kostjukov, A.A. Hernandez Santiago, F.R. Rodriguez, S.R. Castilla, J.A. Parkinson, M.P. Evstigneev, Energetics of ligand binding to the DNA minor groove, Phys. Chem. Chem. Phys. 14 (2012) 5588-5600.

[26] V.V. Kostjukov, N.M. Khomytova, M.P. Evstigneev, Partition of thermodynamic energies of drug-DNA complexation, Biopolymers 91 (2009) 773-790.

[27] V.V. Kostjukov, M.P. Evstigneev, Energy analysis of noncovalent ligand binding to nucleic acids: present and future, Biophysics 59 (2014) 552-555. 
[28] J.B. Chaires, A thermodynamic signature for drug-DNA binding mode, Arch. Biochem. Biophys. 453 (2006) 26-31.

[29] G.P. Brady, K.A. Sharp, Decomposition of interaction free energies in proteins and other complex systems, J. Mol. Biol. 254 (1995) 77-85.

[30] P. Pfeffer, H. Gohlke, DrugScore RNA knowledge-based scoring function to predict RNA-ligand interactions, J. Chem. Inf. Model. 47 (2007) 1868-1876.

[31] C. Guilbert, T.L. James, Docking to RNA via root-mean-square-deviationdriven energy minimization with flexible ligands and flexible targets, J. Chem. Inf. Model. 48 (2008) 1257-1268.

[32] G.E. Kellogg, J.N. Scarsdale, F.A. Fornari, Identification and hydropathic characterization of structural features affecting sequence specificity for doxorubicin intercalation into DNA double-stranded polynucleotides, Nucleic Acids Res. 26 (1998) 4721-4732.

[33] G.E. Kellogg, J.N. Scarsdale, D.J. Cashman, Ligand docking and scoring in DNA oligonucleotides. Binding of doxorubicin and modeled analogs to optimize sequence specificity, Med. Chem. Res. 9 (1999) 592-603.

[34] D.J. Cashman, J.N. Scarsdale, G.E. Kellogg, Hydropathic analysis of the free energy differences in anthracycline antibiotic binding to DNA, Nucleic Acids Res. 31 (2003) 4410-4416.

[35] K.V. Miroshnychenko, A.V. Shestopalova, Flexible docking of DNA fragments and actinocin derivatives, Mol. Simul. 31 (2005) 567-574.

[36] R. Rohs, I. Bloch, H. Sklenar, Z. Shakked, Molecular flexibility in ab initio drug docking to DNA: binding-site and binding-mode transitions in all-atom Monte Carlo simulations, Nucleic Acids Res. 33 (2005) 7048-7057.

[37] J. Dolenc, C. Oostenbrink, J. Koller, W.F. van Gunsteren, Molecular dynamics simulations and free energy calculations of netropsin and distamycin binding to an AAAAA DNA binding site, Nucleic Acids Res. 33 (2005) 725-733.

[38] S.B. Singh, P.A. Kollman, Calculating the absolute free energy of association of netropsin and DNA, J. Am. Chem. Soc. 121 (1999) 3267-3271.

[39] P. Cieplak, S.N. Rao, P.D.J. Grootenhuis, P.A. Kollman, Free energy calculation on base specificity of drug-DNA interactions: application to daunomycin and acridine intercalation into DNA, Biopolymers 29 (1990) 717-727.

[40] A. Mukherjee, R. Lavery, B. Bagchi, J.T. Hynes, On the molecular mechanism of drug intercalation into DNA: a simulation study of the intercalation pathway, free energy, and DNA structural changes, J. Am. Chem. Soc. 130 (2008) 97479755.

[41] W.R. Rudnicki, M. Kurzepa, T. Szczepanik, W. Priebe, B. Lesyng, A simple model for predicting the free energy of binding between anthracycline antibiotics and DNA, Acta Biochim. Pol. 47 (2000) 1-9.

[42] J. Ren, T.C. Jenkins, J.B. Chaires, Energetics of DNA intercalation reactions, Biochemistry 39 (2000) 8439-8447.

[43] F. Leng, J.B. Chaires, M.J. Waring, Energetics of echinomycin binding to DNA, Nucleic Acids Res. 31 (2003) 6191-6197.

[44] I. Haq, J.E. Ladbury, B.Z. Chowdhry, T.C. Jenkins, J.B. Chaires, Specific binding of hoechst 33258 to the d(CGCAAATTTGCG) $)_{2}$ duplex: calorimetric and spectroscopic studies, J. Mol. Biol. 271 (1997) 244-257.

[45] F. Barcelo, D. Capo, J. Portugal, Thermodynamic characterization of the multivalent binding of chartreusin to DNA, Nucleic Acids Res. 30 (2002) 4567-4573.

[46] J. Lah, G. Vesnaver, Energetic diversity of DNA minor-groove recognition by small molecules displayed through some model ligand-DNA systems, J. Mol. Biol. 342 (2004) 73-89.

[47] L. Wang, A. Kumar, D.W. Boykin, C. Bailly, W.D. Wilson, Comparative thermodynamics for monomer and dimer sequence-dependent binding of a heterocyclic dication in the DNA minor groove, J. Mol. Biol. 317 (2002) 361374.

[48] S. Mazur, F.A. Tanious, D. Ding, A. Kumar, D.W. Boykin, I.J. Simpson, S. Neidle, W.D. Wilson, A thermodynamic and structural analysis of DNA minor-groove complex formation, J. Mol. Biol. 300 (2000) 321-337.

[49] M. Baginski, F. Fogolari, J.M. Briggs, Electrostatic and non-electrostatic contributions to the binding free energies of anthracycline antibiotics to DNA, J. Mol. Biol. 274 (1997) 253-267.

[50] P. Kollman, Free energy calculations: applications to chemical and biochemical phenomena, Chem. Rev. 93 (1993) 2395-2417.

[51] W. Wang, O. Donini, C.M. Reyes, P.A. Kollman, Biomolecular simulations: recent developments in force fields, simulations of enzyme catalysis, proteinligand, protein-protein, and protein-nucleic acid noncovalent interactions, Annu. Rev. Biophys. Biomol. Struct. 30 (2001) 211-243.

[52] P.A. Kollman, I. Massova, C. Reyes, B. Kuhn, S. Huo, L. Chong, M. Lee, T. Lee, Y. Duan, W. Wang, O. Donini, P. Cieplak, J. Srinivasan, D.A. Case, T.E. Cheatham, Calculating structures and free energies of complex molecules: combining molecular mechanics and continuum models, Acc. Chem. Res. 33 (2000) 889897.

[53] P.A. Bash, U.C. Singh, R. Langridge, P.A. Kollman, Free energy calculations by computer simulation, Science 236 (1987) 564-568.

[54] H. Gouda, I.D. Kuntz, D.A. Case, P.A. Kollman, Free energy calculations for theophylline binding to an RNA aptamer: comparison of MM-PBSA and thermodynamic integration methods, Biopolymers 68 (2003) 16-34.

[55] C.M. Reyes, P.A. Kollman, Structure and thermodynamics of RNA-protein binding: using molecular dynamics and free energy analyses to calculate the free energies of binding and conformational change, J. Mol. Biol. 297 (2000) $1145-1158$.

[56] B. Jayaram, D. Sprous, D.L. Beveridge, Solvation free energy of biomacromolecules: parameters for a modified generalized born model consistent with the AMBER force field, J. Phys. Chem. B. 102 (1998) 9571-9576.
[57] M. Trieb, C. Rauch, F.R. Wibowo, B. Wellenzohn, K.R. Liedl, Cooperative effects on the formation of intercalation sites, Nucleic Acids Res. 32 (2004) 46964703.

[58] F.A. Tanious, D. Hamelberg, C. Bailly, A. Czarny, D.W. Boykin, W.D. Wilson, DNA sequence dependent monomer-dimer binding modulation of asymmetric benzimidazole derivatives, J. Am. Chem. Soc. 126 (2004) 143153.

[59] W. Treesuwan, K. Wittayanarakul, N.G. Anthony, G. Huchet, G. Alniss, S. Hannongbua, A.I. Khalaf, C.J. Suckling, J.A. Parkinson, S.P. Mackay, A detailed binding free energy study of 2:1 ligand-DNA complex formation by experiment and simulation, Phys. Chem. Chem. Phys. 11 (2009) $10682-$ 10693.

[60] B. Jayaram, K.J. McConnell, S.B. Dixit, D.L. Beveridge, Free energy analysis of protein-DNA binding: the EcoRI endonuclease-DNA complex, J. Comput. Phys. 151 (1999) 333-357.

[61] N. Spackova, T.E. Cheatham, F. Ryjacek, F. Lankas, L. van Meervelt, P. Hobza, J. Sponer, Molecular dynamics simulations and thermodynamics analysis of DNA-drug complexes. Minor groove binding between 4',6-diamidino-2phenylindole and DNA duplexes in solution, J. Am. Chem. Soc. 125 (2003) 1759-1769.

[62] Y. Mu, G. Stock, Conformational dynamics of RNA-peptide binding: a molecular dynamics simulation study, Biophys. J. 90 (2006) 391-399.

[63] M.A. Olson, L. Cuff, Free Energy determinants of binding the rRNA substrate and small ligands to ricin A-chain, Biophys. J. 76 (1999) 28-39.

[64] V.V. Kostyukov, Energy of intercalation of aromatic heterocyclic ligands into DNA and its partition into additive components, Biopolym. Cell 27 (2011) 264-272.

[65] V.V. Kostyukov, Energetics of complex formation of the DNA hairpin structure d (GCGAAGC) with aromatic ligands, Biophysics 56 (2011) 28-39.

[66] V.V. Kostjukov, M.P. Evstigneev, Energy of ligand-RNA complex formation, Biophysics 57 (2012) 450-463.

[67] V.V. Kostjukov, N.M. Khomytova, A.A. Hernandez Santiago, A.-M.C. Tavera, J.S Alvarado, M.P. Evstigneev, Parsing of the free energy of aromatic-aromatic stacking interactions in solution, J. Chem. Thermodyn. 43 (2011) 1424-1434.

[68] J. Dolenc, R. Baron, C. Oostenbrink, J. Koller, W.F. van Gunsteren, Configurational entropy change of netropsin and distamycin upon DNA minor-groove binding, Biophys. J. 91 (2006) 1460-1470.

[69] G. Luck, H. Triebel, M. Warbingi, C. Zimmer, Conformation dependent binding of netropsin and distamycin to DNA and DNA model polymers, Nucleic Acids Res. 1 (1974) 503-530.

[70] C.A. Chang, W. Chen, M.K. Gilson, Ligand configurational entropy and protein binding, Proc. Natl Acad. Sci. U.S.A. 104 (2007) 1534-1539.

[71] S.W. Lee, L. Zhao, A. Pardi, T. Xia, Ultrafast Dynamics Show That the Theophylline and 3-Methylxanthine Aptamers Employ a Conformational Capture Mechanism for Binding Their Ligands, Biochemistry 49 (2010) 2943 2951.

[72] M.P. Latham, G.R. Zimmermann, A. Pardi, NMR chemical exchange as a probe for ligand-binding kinetics in a theophylline-binding RNA aptamer, J. Am. Chem. Soc. 131 (2009) 5052-5053.

[73] D.A. Bondarev, W.J. Skawinski, C.A. Venanzi, Nature of intercalator amiloride-nucelobase stacking. An empirical potential and ab initio electron correlation study, J. Phys. Chem. B 104 (2000) 815-822.

[74] D. Reha, M. Kabelac, F. Ryjacek, J. Sponer, J.E. Sponer, M. Elstner, S. Suhai, P. Hobza, Intercalators. 1. Nature of stacking interactions between intercalators (Ethidium, Daunomycin, Ellipticine, and 4',6-diaminide-2-phenylindole) and DNA base pairs. Ab initio quantum chemical, density functional theory, and empirical potential study, J. Am. Chem. Soc. 124 (2002) 3366-3376.

[75] D.H. Nguyen, T. Dieckmann, M.E. Colvin, W.H. Fink, Dynamics studies of a malachite green-RNA complex revealing the origin of the red-shift and energetic contributions of stacking interactions, J. Phys. Chem. B 108 (2004) 1279-1286.

[76] H.-J. Schneider, Dispersive interactions in solution complexes, Acc. Chem. Res. 48 (2015) 1815-1822.

[77] C.A. Hunter, van der Waals interactions in non-polar liquids, Chem. Sci. 4 (2013) 834-848.

[78] J. Flinders, S.C. DeFina, D.M. Brackett, C. Baugh, C. Wilson, T. Dieckmann, Recognition of planar and nonplanar ligands in the malachite green-RNA aptamer complex, Chem. Biol. Chem. 5 (2004) 62-72.

[79] M.P. Evstigneev, V.V. Kostjukov, A revised treatment of the non-electrostatic contribution to the solvation free energy of DNA-binding ligands, J. Mol. Liq. 163 (2011) 178-180.

[80] S. Wang, T. Peng, C.F. Yang, Investigation on the interaction of DNA and electroactive ligands using a rapid electrochemical method, J. Biochem. Biophys. Methods 55 (2003) 191-204.

[81] M. Aslanoglu, Electrochemical and spectroscopic studies of the interaction of proflavine with DNA, Analyt. Sci. 22 (2006) 439-443.

[82] J. Piehler, A. Brecht, G. Gauglitz, M. Zerlin, C. Maul, R. Thiericke, S. Grabley, Label-free monitoring of DNA-ligand interactions, Analyt. Biochem. 249 (1997) 94-102.

[83] Yu.S. Noskov, C. Lim, Free energy decomposition of protein-protein interactions, Biophys. J. 81 (2001) 737-750.

[84] R.A. Friedman, B. Honig, A free energy analysis of nucleic acid base stacking in aqueous solution, Biophys. J. 69 (1995) 1528-1535.

[85] W.C. Still, A. Tempczyk, R.C. Hawley, T. Hendrickson, Semianalytical treatment of solvation for molecular mechanics and dynamics, J. Am. Chem. Soc. 112 (1990) 6127-6129. 
[86] V.V. Kostjukov, N.M. Khomytova, D.B. Davies, M.P. Evstigneev, Electrostatic contribution to the energy of binding of aromatic ligands with DNA Biopolymers 89 (2008) 680-690.

[87] C. Medhi, J.B.O. Mitchell, S.L. Price, A.B. Tabor, Electrostatic factors in DNA intercalation, Biopolymers 52 (1999) 84-93.

[88] V.V. Kostjukov, O.V. Rogova, M.P. Evstigneev, General features of the energetics of complex formation between ligand and nucleic acids, Biophysics 59 (2014) 546-551.

[89] V.V. Kostjukov, N.M. Khomytova, A.A. Hernandez Santiago, R. Licona Ibarra, D. B. Davies, M.P. Evstigneev, Calculation of the electrostatic charges and energies for intercalation of aromatic drug molecules with DNA, Int. J. Quant Chem. 111 (2011) 711-721.

[90] J.-H. Ha, R.S. Spolar, M.T. Record, Heat capacity and entropy changes in processes involving proteins, Proc. Natl Acad. Sci. U.S.A. 74 (1977) 22362240.

[91] J.-H. Ha, R.S. Spolar, M.T. Record, Role of the hydrophobic effect in stability of site-specific Protein-DNA complexes, J. Mol. Biol. 209 (1989) 801-816.

[92] R.S. Spolar, M.T. Record, Coupling of local folding to site-specific binding of proteins to DNA, Science 263 (1994) 777-784.

[93] R.A. Friedman, B. Honig, The electrostatic contribution to DNA base stacking interactions, Biopolymers 32 (1992) 145-159.

[94] J. Janin, Angstroms and calories, Structure 5 (1997) 473-479.

[95] V.V. Kostjukov, N.M. Khomutova, A.O. Lantushenko, M.P. Evstigneev, Hydrophobic contribution to the free energy of complexation of aromatic ligands with DNA, Biopolym. Cell 25 (2009) 133-141.

[96] G.I. Makhatadze, P.L. Privalov, Energetics of protein structure, Adv. Protein Chem. 47 (1995) 307-425.

[97] Z.S. Derewenda, L. Lee, U. Derewenda, The occurence of C-H...O hydrogen bonds in proteins, J. Mol. Biol. 252 (1995) 248-262.

[98] L.M.F. Holthauzen, J. Rosgen, D.W. Bolen, Hydrogen bonding progressively strengthens upon transfer of the protein urea-denatured state to water and protecting osmolytes, Biochemistry 49 (2010) 1310-1318.

[99] K. Morokuma, Molecular orbital studies of hydrogen bonds. III. CO $\cdots \mathrm{HO}$ hydrogen bond in $\mathrm{H}_{2} \mathrm{CO} \cdots \mathrm{H}_{2} \mathrm{O}$ and $\mathrm{H}_{2} \mathrm{CO} \cdots 2 \mathrm{H}_{2} \mathrm{O}$, J. Chem. Phys. 55 (1971) $1236-1244$.

[100] A.J. Doig, D.H. Williams, Binding energy of an amide-amide hydrogen bond in aqueous and nonpolar solvents, J. Am. Chem. Soc. 114 (1992) 338-343.

[101] V.I. Danilov, V.M. Anisimov, N. Kurita, D. Hovorun, MP2 and DFT studies of the DNA rare base pairs: the molecular mechanism of the spontaneous substitution mutations conditioned by tautomerism of bases, Chem. Phys Lett. 412 (2005) 285-293.

[102] O.V. Shishkin, A. Pelmenschikov, D.M. Hovorun, Molecular structure of free canonical 2'-deoxyribonucleosides: a density functional study, J. Mol. Struct. 526 (2000) 329-341.

[103] Y.P. Yurenko, R.O. Zhurakivsky, S.P. Samijlenko, M. Ghomi, D.M. Hovorun, The whole of intramolecular H-bonding in the isolated DNA nucleoside thymidine. AIM electron density topological study, Chem. Phys. Lett. 447 (2007) 140-146.

[104] S.J. Grabowski, Hydrogen Bonding: New Insights, 2006.

[105] T.C. Jenkins, A.N. Lane, S. Neidle, D.J. Brown, NMR and molecular modeling studies of the interaction of berenil and pentamidine with (CGCAAATTTGCG) $)_{2}$, Eur. J. Biochem. 213 (1993) 1175-1184.

[106] O.P. Boryskina, T.V. Bolbukh, M.A. Semenov, A.I. Gasan, V.Ya. Maleev, Energies of peptide-peptide and peptide-water hydrogen bonds in collagen: evidences from infrared spectroscopy, quartz piezogravimetry and differential scanning calorimetry, J. Mol. Struct. 827 (2007) 1-10.

[107] T.V. Chalikian, Hydrophobic tendencies of polar groups as a major force in molecular recognition, Biopolymers 70 (2003) 492-496.

[108] V.V. Kostjukov, N.M. Khomytova, M.P. Evstigneev, Hydration change on complexation of aromatic ligands with DNA: molecular dynamics simulations, Biopolym. Cell 26 (2010) 36-44.

[109] J.A. Cowan, T. Ohyama, D. Wang, K. Natarajan, Recognition of a cognate RNA aptamer by neomycin B: quantitative evaluation of hydrogen bonding and electrostatic interactions, Nucleic Acids Res. 28 (2000) 2935-2942.

[110] M.M. Islam, S.R. Chowdhury, G.S. Kumar, Spectroscopic and calorimetric studies on the binding of alkaloids berberine, palmatine and coralyne to double stranded RNA polynucleotides, J. Phys. Chem. B 113 (2009) 1210 1224.

[111] P. Kovacic, L.P.G. Wakelin, DNA molecular electrostatic potential: novel perspectives for the mechanism of action of anticancer drugs involving electron transfer and oxidative stress, Anticancer Drug Des. 16 (2001) 175184.

[112] T. Kubar, M. Hanus, F. Ryjacek, P. Hobza, Binding of cationic and neutral phenanthridine intercalators to a DNA oligomer is controlled by dispersion energy: quantum chemical calculations and molecular mechanics simulations, Chem. Eur. J. 12 (2006) 280-290.

[113] A. Szent-Gyorgyi, I. Isenberg, On the electron-donating properties of indoles, Proc. Natl Acad. Sci. U.S.A. 49 (1960) 1334-1336.

[114] T. Sakurai, H. Hosoya, Charge-transfer complexes of nicotinamide-adenine dinucleotide analogues and flavin mononucleotide, Biochim. Biophys. Acta 112 (1966) 459-468.

[115] J. Molnar, S. Foldeak, M.J. Nakamura, F. Gaizer, F. Gutman, The influence of charge transfer complex formation on the antibacterial activity of some tricyclic drugs, Xenobiotica 21 (1991) 309-316.
[116] L.M. Du, A.P. Lin, Y.Q. Yang, Spectrofluorimetric determination of certain fluoroquinolone through charge transfer complex formation, Analyt. Lett. 37 (2004) 2175-2188.

[117] S. Tsuzuki, K. Honda, T. Uchimaru, M. Mikami, Intermolecular interactions of nitrobenzene-benzene complex and nitrobenzene dimer: significant stabilization of slipped-parallel orientation by dispersion interaction, J. Chem. Phys. 125 (2006) 124304

[118] K.E. Riley, P. Hobza, On the importance and origin of aromatic interactions in chemistry and biodisciplines, Acc. Chem. Res. 46 (2013) 927-936.

[119] C.A. Hunter, Arene-arene interactions: electrostatic or charge transfer?, Angew Chem. Int. Ed. 32 (1993) 1584-1586.

[120] M.P. Evstigneev, Hetero-association of aromatic molecules in aqueous solution, Int. Rev. Phys. Chem. 33 (2014) 229-273.

[121] H. Kamisetti, A. Ramanathan, C. Bailey-Kellogg, C.J. Langmead, Accounting for conformational entropy in predicting binding free energies of protein-protein interactions, Proteins: Struct. Funct., Bioinform. 79 (2011) 444-462.

[122] H.-X. Zhou, M.K. Gilson, Theory of free energy and entropy in noncovalent binding, Chem. Rev. 109 (2009) 4092-4107.

[123] M. Kolar, T. Kubar, P. Hobza, Sequence-dependent configurational entropy change of DNA upon intercalation, J. Phys. Chem. B 114 (2010) 13446-13454.

[124] A.V. Finkelstein, J. Janin, The price of lost freedom: entropy of bimolecular complex formation, Protein Eng. 3 (1989) 1-3.

[125] J. Janin, C. Chothia, Role of hydrophobicity in the binding of coenzymes. Appendix: translational and rotational contribution to the free energy of dissociation, Biochemistry 17 (1978) 2943-2948.

[126] M.K. Gilson, J.A. Given, B.L. Bush, J.A. McCammon, The statisticalthermodynamic basis for computation of binding affinities: a critical review, Biophys. J. 72 (1997) 1047-1069.

[127] H. Wang, C.A. Laughton, Molecular modelling methods for prediction of sequence-selectivity in DNA recognition, Methods 42 (2007) 196-203.

[128] S.A. Harris, E. Gavathiotis, M.S. Searle, M. Orozco, C.A. Laughton, Cooperativity in drug-DNA recognition: a molecular dynamics study, J. Am. Chem. Soc. 123 (2001) 12658-12663.

[129] J. Schlitter, Estimation of absolute and relative entropies of macromolecules using the covariance matrix, Chem. Phys. Lett. 215 (1993) 617-621.

[130] C.E. Bostock-Smith, S.A. Harris, C.A. Laughton, M.S. Searle, Induced fit DNA recognition by a minor groove binding analogue of Hoechst 33258: fluctuations in DNA A tract structure investigated by NMR and molecular dynamics simulations, Nucleic Acids Res. 29 (2001) 693-702.

[131] M.K. Gilson, H.-X. Zhou, Calculation of protein-ligand binding affinities, Annu. Rev. Biophys. Biomol. Struct. 36 (2007) 21-42.

[132] R. Nifosi, C.M. Reyes, P.A. Kollman, Molecular dynamics studies of the HIV-1 TAR and its complex with argininamide, Nucleic Acids Res. 28 (2000) 4944-4955.

[133] C. Ma, N.A. Baker, S. Joseph, J.A. McCammon, Binding of aminoglycoside antibiotics to the small ribosomal subunit: a continuum electrostatics investigation, J. Am. Chem. Soc. 124 (2002) 1438-1442.

[134] C.M. Reyes, R. Nifosi, A.D. Frankel, P.A. Kollman, Molecular dynamics and binding specificity analysis of the bovine immunodeficiency virus BIV TatTAR complex, Biophys. J. 80 (2001) 2833-2842.

[135] M.A. Olson, Calculations of free-energy contributions to protein-RNA complex stabilization, Biophys. J. 81 (2001) 1841-1853.

[136] V.V. Kostyukov, N.M. Khomutova, M.P. Evstigneev, Contribution of changes in translational, rotational, and vibrational degrees of freedom to the energy of complex formation of aromatic ligands with DNA, Biophysics 54 (2009) 606615.

[137] S.K. Pal, L. Zhao, A.H. Zewail, Water at DNA surfaces: Ultrafast dynamics in minor groove recognition, Proc. Natl Acad. Sci. U.S.A. 100 (2003) 8113-8118.

[138] M.P. Evstigneev, A.O. Lantushenko, I.V. Golovchenko, Hidden entropic contribution in the thermodynamics of molecular complexation, Phys. Chem. Chem. Phys. 18 (2016) 7617-7625.

[139] V.V. Kostjukov, M.P. Evstigneev, Relation between the change in DNA elasticity on ligand binding and the binding energetics, Phys. Rev. E 86 (2012) 031919.

[140] D.A. Beshnova, A.O. Lantushenko, M.P. Evstigneev, Does the ligandbiopolymer equilibrium binding constant depend on the number of bound ligands?, Biopolymers 93 (2010) 932-935

[141] P.L.F. de Castro, M. Zacharias, DAPI binding to the DNA minor groove: a continuum solvent analysis, J. Mol. Recognit. 15 (2002) 209-220.

[142] J. Dolenc, U. Borstnik, M. Hodoscek, J. Koller, D. Janezic, An ab initio QM/MM study of the conformational stability of complexes formed by netropsin and DNA. The importance of van der Waals interactions and hydrogen bonding, J. Mol. Struct. 718 (2005) 77-85.

[143] B. Tidor, M. Karplus, The contribution of vibrational entropy to molecular association: the dimerization of insulin, J. Mol. Biol. 238 (1994) 405-414.

[144] K. Gallagher, K. Sharp, Electrostatic contributions to heat capacity changes of DNA-ligand binding, Biophys. J. 75 (1998) 769-776.

[145] V.V. Kostyukov, N.M. Khomutova, M.P. Evstigneev, Contribution of enthalpy to the energetics of complex formation of aromatic ligands with DNA Biophysics 56 (2011) 634-643.

[146] C.A. Hunter, Quantifying intermolecular interactions: guidelines for the molecular recognition toolbox, Angew. Chem. Int. Ed. 43 (2004) 5310-5324.

[147] V. Maleev, M. Semenov, E. Kruglova, T. Bolbukh, A. Gasan, E. Bereznyak, A Shestopalova, Spectroscopic and calorimetric study of DNA interaction with a new series of actinocin derivatives, J. Mol. Struct. 645 (2003) 145-158. 
[148] L.A. Marky, R.B.J. Macgregor, Hydration of dA.dT polymers: role of water in the thermodynamics of ethidium and propidium intercalation, Biochemistry 29 (1990) 4805-4811.

[149] F. Han, T.V. Chalikian, Hydration changes accompanying nucleic acid intercalation reactions: volumetric characterizations, J. Am. Chem. Soc. 125 (24) (2003) 7219-7229.

[150] X. Shi, R.B.J. Macgregor, Volume and hydration changes of DNA-ligand interactions, Biophys. Chem. 125 (2007) 471-482.

[151] T.V. Chalikian, K.J. Breslauer, Volumetric properties of nucleic acids, Biopolymers 48 (1998) 264-280.

[152] H.J. Yu, J.S. Ren, J.B. Chaires, X.G. Qu, Hydration of drug-DNA complexes: greater water uptake for adriamycin compared to daunomycin, J. Med. Chem. 51 (2008) 5909-5911.

[153] X. Qu, J.B. Chaires, Hydration changes for DNA intercalation reactions, J. Am. Chem. Soc. 123 (2001) 1-3.

[154] N.Y. Sidorova, D.C. Rau, The osmotic sensitivity of netropsin analog binding to DNA, Biopolymers 35 (1995) 377-384.
[155] N.N. Degtyareva, B.D. Wallace, A.R. Bryant, K.M. Loo, J.T. Petty, Hydration changes accompanying the binding of minor groove ligands with DNA, Biophys. J. 92 (2007) 959-965.

[156] A.V. Shestopalova, D.A. Pesina, V.A. Kashpur, O.V. Khorunzhaya, Hydration of DNA-binding biological active compounds: EHF dielectrometry and molecular modeling results, Struct. Chem. 27 (2016) 159-173.

157] S. Shimizu, Estimating hydration changes upon biomolecular reactions from osmotic stress, high pressure, and preferential hydration experiments, Proc. Natl Acad. Sci. U.S.A. 101 (2004) 1195-1199.

[158] F. Han, N. Taulier, T.V. Chalikian, Association of the minor groove binding drug hoechst 33258 with d(CGCGAATTCGCG)2: volumetric, calorimetric, and spectroscopic characterizations, Biochemistry 44 (2005) 9785-9794.

JCT 2019-461 